\title{
Gene expression analysis of human induced pluripotent stem cell-derived neurons carrying copy number variants of chromosome $15 q 11-q 13.1$
}

Noelle D Germain ${ }^{1}$, Pin-Fang Chen ${ }^{1}$, Alex M Plocik', Heather Glatt-Deeley ${ }^{1}$, Judith Brown ${ }^{2}$, James J Fink ${ }^{3}$, Kaitlyn A Bolduc ${ }^{3}$, Tiwanna M Robinson ${ }^{3}$, Eric S Levine ${ }^{3}$, Lawrence T Reiter ${ }^{4}$, Brenton R Graveley ${ }^{1,5}$, Marc Lalande ${ }^{1}$ and Stormy J Chamberlain ${ }^{1^{*}}$

\begin{abstract}
Background: Duplications of the chromosome 15q11-q13.1 region are associated with an estimated 1 to 3\% of all autism cases, making this copy number variation (CNV) one of the most frequent chromosome abnormalities associated with autism spectrum disorder (ASD). Several genes located within the 15q11-q13.1 duplication region including ubiquitin protein ligase E3A (UBE3A), the gene disrupted in Angelman syndrome (AS), are involved in neural function and may play important roles in the neurobehavioral phenotypes associated with chromosome 15q11-q13.1 duplication (Dup15q) syndrome.
\end{abstract}

Methods: We have generated induced pluripotent stem cell (iPSC) lines from five different individuals containing CNVs of 15q11-q13.1. The iPSC lines were differentiated into mature, functional neurons. Gene expression across the 15q11-q13.1 locus was compared among the five iPSC lines and corresponding iPSC-derived neurons using quantitative reverse transcription PCR (qRT-PCR). Genome-wide gene expression was compared between neurons derived from three iPSC lines using mRNA-Seq.

Results: Analysis of 15q11-q13.1 gene expression in neurons derived from Dup15q iPSCs reveals that gene copy number does not consistently predict expression levels in cells with interstitial duplications of 15q11-q13.1. mRNA-Seq experiments show that there is substantial overlap in the genes differentially expressed between 15q11-q13.1 deletion and duplication neurons, Finally, we demonstrate that UBE3A transcripts can be pharmacologically rescued to normal levels in iPSC-derived neurons with a 15q11-q13.1 duplication.

Conclusions: Chromatin structure may influence gene expression across the 15q11-q13.1 region in neurons. Genome-wide analyses suggest that common neuronal pathways may be disrupted in both the Angelman and Dup15q syndromes. These data demonstrate that our disease-specific stem cell models provide a new tool to decipher the underlying cellular and genetic disease mechanisms of ASD and may also offer a pathway to novel therapeutic intervention in Dup15q syndrome.

Keywords: UBE3A, autism, induced pluripotent stem cells, 15q duplication, Angelman syndrome

\footnotetext{
* Correspondence: chamberlain@uchc.edu

'Department of Genetics and Developmental Biology, University of

Connecticut Health Center, 400 Farmington Avenue, Farmington, CT 06032,

USA

Full list of author information is available at the end of the article
} 


\section{Background}

One of the most frequent chromosome anomalies associated with autism is the duplication of chromosome 15q11-q13.1 [1-8]. The parent-of-origin is an important factor for chromosome 15q11-q13.1 duplication (Dup15q) syndrome because the chromosome $15 \mathrm{q} 11-\mathrm{q} 13.1$ region is subject to genomic imprinting, which is an epigenetic process that results in monoallelic gene expression. The 15q11-q13.1 duplications that lead to autism are most frequently of maternal origin. In addition to autism, individuals with maternally-inherited or derived duplications of chromosome 15q11-q13.1 have hypotonia, developmental delay, speech and language delay, behavioral difficulties, and seizures. There are two major classes of chromosomal duplication. First, interstitial duplications (int dup(15)) result in tandem copies of maternal 15q11-q13.1 lying in a head-to-head orientation on the same chromosome arm. Second, isodicentric chromosome 15 (idic(15)) duplications result in two additional copies of maternal 15q11-q13.1 which are flanked by two centromeres on a supernumerary chromosome. Not surprisingly, individuals with idic(15), who have 4 copies of $15 \mathrm{q} 11-\mathrm{q} 13.1$, are more severely affected than those with int dup(15), who have 3 copies [9].

Deletion of the maternal allele of chromosome 15q11q13.1 results in Angelman syndrome (AS) a neurodevelopmental disorder characterized by developmental delay, absent speech and seizures [10]. Deletion or loss of function of a single maternally expressed gene within 15q11q13.1, encoding ubiquitin protein ligase E3A (UBE3A), is sufficient to cause AS [11-13]. Several studies suggest that maternal duplication of $U B E 3 A$ underlies the autism phenotype associated with 15q11-q13.1 duplications $[1,2,8,14]$. However, the duplicated region also includes several other genes, including a cluster of genes encoding gamma aminobutyric acid (GABA) receptor subunits, GABRB3, GABRG3, and GABRA5. Additionally, all idic(15) duplications and some int $\operatorname{dup}(15)$ duplications include cytoplasmic FMRP interacting protein (CYFIP1), which binds to and antagonizes the fragile $\mathrm{X}$ mental retardation protein (FMRP) [15], the protein whose loss of function causes Fragile $\mathrm{X}$ syndrome; and nonimprinted in Prader-Willi/ Angelman syndrome region 1 and 2 (NIPA1 and NIPA2), genes encoding putative magnesium transporters involved in seizures, schizophrenia, and hereditary spastic paraplegia $[16,17]$. Therefore, non-imprinted genes in the $15 q$ critical duplication locus may play an important role in the phenotype of individuals with both int dup (15) and idic(15).

Here we report the generation of induced pluripotent stem cell (iPSC) lines and neurons from individuals with both isodicentric and interstitial duplications of chromosome 15q11-q13.1. We compared gene expression between iPSCs and iPSC-derived neurons with both deletions and duplications of this region. We found that while the overall gene expression levels of the chromosome 15q genes largely reflect the copy number in AS and idic(15) iPSCs and neurons, the gene expression levels did not correlate as well with copy number in the paternal or maternal int dup(15) neurons, suggesting that the inverted duplication may disrupt distal regulatory elements that act primarily in neural tissue. We also compared global transcriptome expression between AS and idic(15) neurons and found that despite having opposite genetic anomalies (deletion in AS and duplication in idic(15)), most of the genes differentially expressed in both disorders were changed in the same direction. In fact, both disorders result in the downregulation of genes involved in neuron development, including many autism candidate genes. Together, these data suggest different patterns of neuronal gene regulation between int $\operatorname{dup}(15)$ and idic(15) and similar neuronal pathways disrupted in deletions and duplication of chromosome 15q11-q13.1.

\section{Methods}

Patient samples, regulatory approvals, iPSC derivation, and cell culture

Idic(15) fibroblasts (catalog ID: GM07992) were obtained from the Coriell Institute for Medical Research Cell Repository. Idic(15) umbilical cord blood cells were donated by the patient's family through the Dup $15 q$ Alliance and were exempted from consideration as human subject research by the University of Connecticut Health Center Institutional Review Board (IRB). Fibroblasts from an individual with inherited paternal interstitial 15q11-q13.1 duplication (patient 801-015) and an individual mosaic for maternal interstitial 15q11-q13.1 duplication (mother of patient 801-018) were obtained by Dr. Lawrence $\mathrm{T}$. Reiter under IRB approval number 11-01350-FB from the University of Tennessee Health Science Center. All patient samples were obtained from subjects after they had given informed consent and were subsequently de-identified.

iPSCs were generated with Institutional Biosafety Committee approval number IBC08-005 from the University of Connecticut Health Center by the University of Connecticut - Wesleyan University Stem Cell Core as previously reported [18]. iPSCs were maintained on irradiated mouse embryonic fibroblasts (MEFs) in human embryonic stem cell medium which consists of DMEM/ F12, 20\% knockout serum replacement, $1 \mathrm{mM}$ L-glutamine, $1 \mathrm{X}$ nonessential amino acids, $100 \mathrm{mM} \beta$-mercaptoethanol (all Gibco products through Life Technologies, Grand Island, NY, USA), and $4 \mathrm{ng} / \mathrm{mL}$ basic fibroblast growth factor (bFGF, Millipore, Billerica, MA, USA). iPSCs were manually passaged every 6 or 7 days. 


\section{Karyotype analysis, DNA fluorescence in situ hybridization, and whole genome copy number variation analysis}

Cytogenetic analysis of Dup15q iPSCs was performed by the Genetics and Genomics Division of the University of Connecticut - Wesleyan University Stem Cell Core. Twenty G-banded metaphase cells from each iPSC line were examined to generate a karyotype for each line. DNA fluorescence in situ hybridization (FISH) was performed on both metaphase and interphase cells using a dual-labeled probe containing the small nuclear ribonucleoprotein polypeptide $\mathrm{N}(S N R P N)$ gene and a control locus at 15qter (Cytocell Aquarius LPU005-A-034359, Cytocell, Cambridge, UK). Whole genome copy number analysis was performed on genomic DNA isolated from AS del 1-0 and Idic1-8 iPSCs using the Affymetrix CytoScan HD Array (Affymetrix, Santa Clara, CA, USA) to determine deletion/duplication breakpoints. Duplication breakpoints for the maternal and paternal Int dup (15) patient samples were previously published (as patients 801-018 and 801-015, respectively) [8] and for IdicCB were provided in an array $\mathrm{CGH}$ report accompanying the patient sample.

\section{Neural differentiation}

iPSC-derived neural progenitors were generated by either embryoid body (EB)-based or monolayer differentiation according to established protocols $[19,20]$ with minor modifications. For EB differentiation, iPSC colonies were manually detached from MEF feeders and put into suspension culture rather than enzymatic dissociation. After three weeks of neural differentiation (in both protocols), neural progenitors were plated on poly-ornithine/laminin coated substrates in neural differentiation medium consisting of Neurobasal Medium, B-27 supplement, nonessential amino acids, and L-glutamine (all Gibco products through Life Technologies, Grand Island, NY, USA) supplemented with $1 \mu \mathrm{M}$ ascorbic acid, $200 \mu \mathrm{M}$ cyclic adenosine monophosphate (cAMP), $10 \mathrm{ng} / \mathrm{mL}$ brainderived neurotrophic factor (BDNF, Peprotech, Rocky Hill, NJ, USA), and $10 \mathrm{ng} / \mathrm{mL}$ glial-derived neurotrophic factor (GDNF, Peprotech, Rocky Hill, NJ, USA). All experiments were conducted on neural cultures that were at least 10 weeks old.

For mithramycin experiments, mithramycin A (SigmaAldrich, St. Louis, MO, USA) was prepared as a $1 \mathrm{mM}$ concentrated stock in dimethyl sulfoxide (DMSO, SigmaAldrich, St. Louis, MO, USA). Working stocks were diluted in DMSO and added to neural differentiation medium immediately before addition to neural cultures.

\section{Immunocytochemistry}

Immunocytochemistry was performed as previously described [18] on iPSCs or 10-week old iPSC-derived neurons that had been cultured on glass coverslips. The following antibodies and concentrations were used: mouse anti-Tra-1-60 (1:200, Santa Cruz Biotechnology, Inc, Dallas, TX, USA), Nanog (1:200, Abcam, Cambridge, UK), mouse anti-SSEA-4 (1:20, Developmental Studies Hybridoma Bank, Iowa City, IA, USA), chicken antiMAP2 (1:10,000, Abcam, Cambridge, UK), rabbit antiMAP2 (1:500, Millipore, Billerica, MA, USA), rabbit anti-Synapsin I (1:400, Millipore, Billerica, MA, USA), mouse anti-PSD-95 (1:100, NeuroMab, Davis, CA, USA), rabbit anti-S100ß (1:200, Abcam, Cambridge, UK), mouse anti-VGlut1 (1:100, Synaptic Systems, Gottingen, Germany), and rabbit anti-Gad65 (1:500, Sigma-Aldrich, St. Loius, MO, USA). All AlexaFluor fluorochrome conjugated (488, 594, and 647) secondary antibodies (Life Technologies, Grand Island, NY, USA) were used at 1:400. A goat anti-chicken IgY-650 secondary antibody (Abcam, Cambridge, UK) was used at 1:250. Nuclei were counterstained with DAPI and coverslips were mounted on slides with Vectashield (Vector Laboratories, Burlingame, CA, USA). Slides were imaged using a Zeiss Axiovision microscope (Carl Zeiss, Germany) at 20X, 40X, and 63X magnification.

\section{Chromatin immunoprecipitation}

Chromatin immunoprecipitation (ChIP) was performed according to the EZ-Magna ChIP protocol (Millipore, Billerica, MA, USA) according to manufacturer's instructions with minor modifications. DMSO or mithramycintreated 10-week old idic(15) neurons were treated with $1 \%$ formaldehyde to crosslink DNA/protein. Instead of a two-step cell lysis, cells were lysed once for 15 minutes in an SDS Cell Lysis Buffer (Millipore, Billerica, MA, USA) before sonication. A rabbit polyclonal anti-YY-1 (sc-281, Santa Cruz Biotechnology, Inc, Dallas, TX, USA) was used at $5 \mu \mathrm{g}$ per immunoprecipitation reaction. For ChIP analysis of Sp1 binding, the following antibodies were used: mouse monoclonal anti-Sp1 (sc-17824, Santa Cruz Biotechnology, Inc, Dallas, TX, USA) and anti-Sp1 (\#9389, Cell Signaling Technology, Beverly, MA, USA). Novex Protein A DynaBeads magnetic beads (Life Technologies, Grand Island, NY, USA) were used during immunoprecipitation. Immunoprecipitated DNA was purified by phenol-chloroform extraction and used for quantitative polymerase chain reaction (qPCR) with SYBR-Green reagents (Life Technologies, Grand Island, NY, USA). The following primers were used for qPCR: UBE3A-exon1 forward GGC AGA GGT GAA GCG TAA GT, $U B E 3 A$-exon1 reverse AGA TCC GTG TGT CTC CCA AG, UBE3A-upstream forward TCT GTG ACC CGA AAG AAT AAA CC, UBE3A-upstream reverse TTC CTC TGC TGG GTA CAC CAA. Sp1 promoter forward TGC CCG CCT GAT TTC TGA, Sp1 promoter reverse GGA TAT GCT TGG GCA AAA 
TCC, DHFR promoter forward TCG CCT GCA CAA ATA GGG AC, DHFR promoter reverse AGA ACG CGC GGT CAA GTT TG. ChIP was performed with triplicate independent batches of neurons. qPCR was performed in triplicate for each DNA sample and $\mathrm{Ct}$ values were used to calculate percent of input. Percent of input values for YY-1 binding were normalized by subtracting the background binding of normal rabbit IgG (Millipore, Billerica, MA, USA). Fold enrichment was then calculated by dividing percent input in mithramycintreated samples by percent input of DMSO-treated controls.

\section{Quantitative reverse-transcription PCR}

Total RNA was isolated from iPSCs, EBs, or iPSC-derived neurons using RNA-Bee (AMS Biotechnology, Lake Forest, CA, USA) according to the manufacturer's protocol. cDNA was produced using the High Capacity cDNA Reverse Transcription Kit (Life Technologies, Grand Island, NY, USA).

Analysis of iPSC pluripotency genes was performed on independent cultures of each iPSC line in triplicate with a TaqMan Human Stem Cell Pluripotency Array (Life Technologies, Grand Island, NY, USA). Analysis of multipotency and neural differentiation capacity was performed with a custom TaqMan Gene Signature Array Card (Life Technologies, Grand Island, NY, USA) as previously described [21] on three independent batches of EBs derived from each iPSC line. This custom array includes representative genes from all three germ lineages as well as pluripotency genes. A full list of gene assays included in the custom array is available in Martins-Taylor et al. [21]. Ct values for all genes were first normalized to $18 \mathrm{~S}$ rRNA and then set relative to the housekeeping gene glyceraldehyde-3-phosphate dehydrogenase (GAPDH).

Analysis of 15q11-q13.1 genes and selected autism candidate genes in iPSCs and iPSC-derived neurons was performed in duplicate or triplicate from independent cultures. All qPCR assays used were TaqMan Gene Expression Assays (Life Technologies, Grand Island, NY, USA). Ct values for each gene were normalized to the house keeping gene GAPDH. Relative expression was quantified as $2^{\wedge^{-\Delta \Delta C t}}$ using normal (Nml 1-0) iPSCs or normal iPSC-derived neurons as the calibrator sample.

\section{Methylation analysis}

Analysis of methylation at the Prader-Willi syndrome imprinting center (PWS-IC) was performed using the Methyl-Profiler DNA Methylation qPCR Assay kit (SA Biosciences, Valencia, CA, USA) and the EpiTect Methyl qPCR Assay for SNRPN (Qiagen, Valencia, CA, USA) as previously described [18].

\section{Allele-specific single nucleotide polymorphism analysis}

The UCSC Genome Browser (http://genome.ucsc.edu/) was used to identify a single nucleotide polymorphism (SNP), rs691, which is located in the last exon of the imprinted in Prader-Willi syndrome $(I P W)$ gene. Genomic DNA from mat. int dup(15), pat. int dup(15), and idic(15) fibroblasts, and IdicCB-09 iPSCs was used to determine heterozygosity for rs691 by PCR amplification across the SNP followed by sequencing by GENEWIZ. Total RNA was isolated from fibroblasts (mat. int $\operatorname{dup}(15)$ and idic(15)), iPSCs, and iPSC-derived neurons (mat. int dup (15)-02, pat. int dup(15)-04, Idic1-8, and IdicCB-09) using RNA-Bee (AMS Biotechnology, Lake Forest, CA, USA) and DNase treated using Turbo DNA-free Kit (Life Technologies, Grand Island, NY, USA). cDNA was generated using the High Capacity cDNA Reverse Transcription Kit (Life Technologies, Grand Island, NY, USA) and used for PCR amplification across rs691 followed by sequencing by GENEWIZ. The following primers were used to amplify across rs691: forward ATG CCC TCC TCT CTT CCA AT and reverse ATA GGG AGG TTC ATT GCA CA.

\section{Electrophysiology}

Individual coverslips were transferred to a recording chamber (room temperature) fixed to the stage of an Olympus BX51WI microscope (Olympus, Tokyo, Japan) fitted with a 40x water-immersion lens. The recording chamber was continuously perfused at $2 \mathrm{ml} / \mathrm{min}$ with oxygenated artificial cerebrospinal fluid $(125 \mathrm{mM} \mathrm{NaCl}$, $2.5 \mathrm{mM} \mathrm{KCl}, 1.25 \mathrm{mM} \mathrm{NaH} 2 \mathrm{PO} 4,1 \mathrm{mM} \mathrm{MgCl} 2-6 \mathrm{H} 2 \mathrm{O}$, $25 \mathrm{mM} \mathrm{NaHCO} 3,2 \mathrm{mM} \mathrm{CaCl} 2$, and $25 \mathrm{mM}$ dextrose). Cells were selected for recording based on neuronal morphology. Whole-cell voltage clamp (holding potential $=-70 \mathrm{mV}$ ) and current clamp recordings were conducted as previously described $[18,22]$. Series resistance (Rs) was compensated to $70 \%$ or greater at 10 to $100 \mu \mathrm{s}$ lag. During the course of the experiments, input resistance (Ri) was continuously monitored with $5 \mathrm{mV}$ hyperpolarizing voltage steps $(50 \mathrm{~ms})$. Neurons were rejected from analyses if (1) Rs was $>25$ MOhms at the time of break-in, if (2) Ri changed by $>15 \%$ during the course of an experiment, or if (3) Ri fell below 100 MOhms.

\section{Transcriptome analysis}

Total RNA was isolated from two biological replicates of 10-week-old neurons derived from AS (AS del 1- 0), normal (Nml 1- 0), and idic(15) (Idic1-8) iPSCs. mRNASeq libraries were prepared using $5 \mu \mathrm{g}$ of total RNA according to manufacturer's specifications (Illumina, San Diego, CA, USA) using the Paired-end Library kit as described in [23]. Libraries were multiplexed and sequenced on Illumina GAIIx and HiSeq 2000 sequencers (Illumina, San Diego, CA, USA). The reads were aligned to the human genome (hg19) using Bowtie (version 0.12.0-beta1) 
and Tophat (version 1.3.1) [24]. At least 20 million mapped reads were generated for each sample. Cufflinks (version 0.9.3) was used to quantitate expression levels for all hg19 UCSC genes and transcripts as fragments per kilobase gene model per million base pairs (FPKM) [25]. All raw sequencing data has been deposited in the National Center for Biotechnology Information (NCBI) Sequence Read Archive (http://www.ncbi.nlm.nih.gov/sra) under the accession number SRP044749.

\section{Copy number analysis}

The copy number of $U B E 3 A$ in each iPSC line was analyzed by qPCR using genomic DNA purified from two biological replicates and TaqMan Copy Number Assays (Hs01665678_cn and Hs03908756_cn, Life Technologies, Grand Island, NY, USA). The RNase P Copy Number Reference Assay was used as an endogenous reference gene to allow for quantification of $U B E 3 A$ copy number. Data were analyzed using the CopyCaller v2.0 software from Applied Biosystems (Life Technologies, Grand Island, NY, USA).

\section{RNA FISH}

iPSCs grown on glass coverslips were processed for RNA-FISH as previously described [21] with the following modifications: UBE3A BAC probe RP11-1081A4 was labeled by nick translation and $500 \mathrm{ng}$ of labeled probe was used for hybridization.

\section{Statistical analyses}

Electrophysiology data for current-voltage relationship and average frequency and amplitude of synaptic currents are presented as the mean plus or minus the standard error of the mean. All qPCR data were analyzed using Microsoft Excel and are presented as the mean relative expression plus or minus the standard error of the mean. Differential gene expression was analyzed for statistical significance using either one-way ANOVA followed by Tukey's multiple comparisons test or an unpaired twotailed $t$-test. ChIP data are presented as the mean fold enrichment plus or minus the standard error of the mean. Differences in fold enrichment were analyzed for statistical significance using an unpaired two-tailed $t$-test.

\section{Results}

\section{Generation of iPSCs from Dup15q patients}

iPSC lines were established using fibroblasts from one idic (15) individual, one individual with a paternally-inherited duplication of chromosome 15q11-q13.1, and one individual who was mosaic for a maternally-inherited interstitial duplication of chromosome 15q11-q13.1 (Figure 1a). iPSC lines were also established from a cord blood sample from one individual with idic(15) (Figure 1a). iPSCs were generated using either retroviral, lentiviral, or episomal vectors encoding POU class 5 homeobox 1 (OCT4), SRY-box 2 (SOX2), Kruppel-like factor 4 (KLF4), v-myc avian myelocytomatosis viral oncogene homolog $(M Y C)$, and lin28 homolog A (LIN28) [see Additional file 1: Table S1] [26-28]. Reprogrammed colonies were initially identified morphologically, and were subsequently validated using qRT-PCR and/or immunocytochemistry to verify the expression of the pluripotency markers, NANOG, stage specific embryonic antigen 4 (SSEA4), and TRA1-60 (Figure 1b-d) and pluripotency genes [see Additional file 2: Figure S1A]. iPSCs were shown to have the expected karyotypes of 46, XX (normal); 46,XX.ish $\operatorname{dup}(15)(\mathrm{q} 11.2 \mathrm{q} 11.2)(\mathrm{SNRPN}++)$; or $47, \mathrm{XX},+$ idic(15).ish15q12 SNRPN x 4, 15qter X2 (Figure 1e). Interstitial duplication of 15q11-q13.1 or the presence of the isodicentric chromosome 15 was confirmed in representative iPSC clones by DNA FISH with a probe for the SNRPN gene and a control probe in the distal long arm of chromosome 15 (Figure 1f and Figure 1g). Breakpoints involved in the duplications in the two int $\operatorname{dup}(15)$ samples were previously identified by array comparative genomic hybridization (CGH) [8]. Breakpoints in the cord blood idic(15) sample were determined by genetic report obtained during diagnosis, and the breakpoints in the fibroblast idic(15) and AS samples were determined using a high density SNP array as part of the current study. Gene expression arrays were used to analyze gene expression in iPSC-derived EBs after 16 days of spontaneous differentiation as previously described $[18,21]$. These data demonstrate that all iPSCs used are capable of multi-lineage differentiation, since early lineage markers representing each of the three embryonic germ layers as well as the trophectoderm layer are expressed [see Additional file 2: Figure S1B].

\section{Dup15q iPSCs maintain the appropriate methylation imprint following reprogramming}

We previously demonstrated that the methylation imprint at the PWS-IC was maintained during reprogramming in AS, PWS and normal iPSCs [18]. We assessed the methylation imprint at the PWS-IC in normal and Dup15q iPSCs using methylation-specific qPCR $[29,30]$. We expected that iPSCs from idic(15) iPSCs would have approximately $75 \%$ methylation due to the presence of 3 maternal and 1 paternal alleles. We expected that paternal int dup(15) iPSCs would have approximately 33\% methylation resulting from one maternal and two paternal alleles. We expected that some of the iPSCs from the individual mosaic for a maternal int dup(15) would arise from cells with the duplication and thus would have $66 \%$ methylation resulting from 2 maternal and one paternal alleles, while others would be derived from the normal cells and have 50\% methylation. Consistent with this prediction, idic(15) iPSCs had methylation levels ranging 


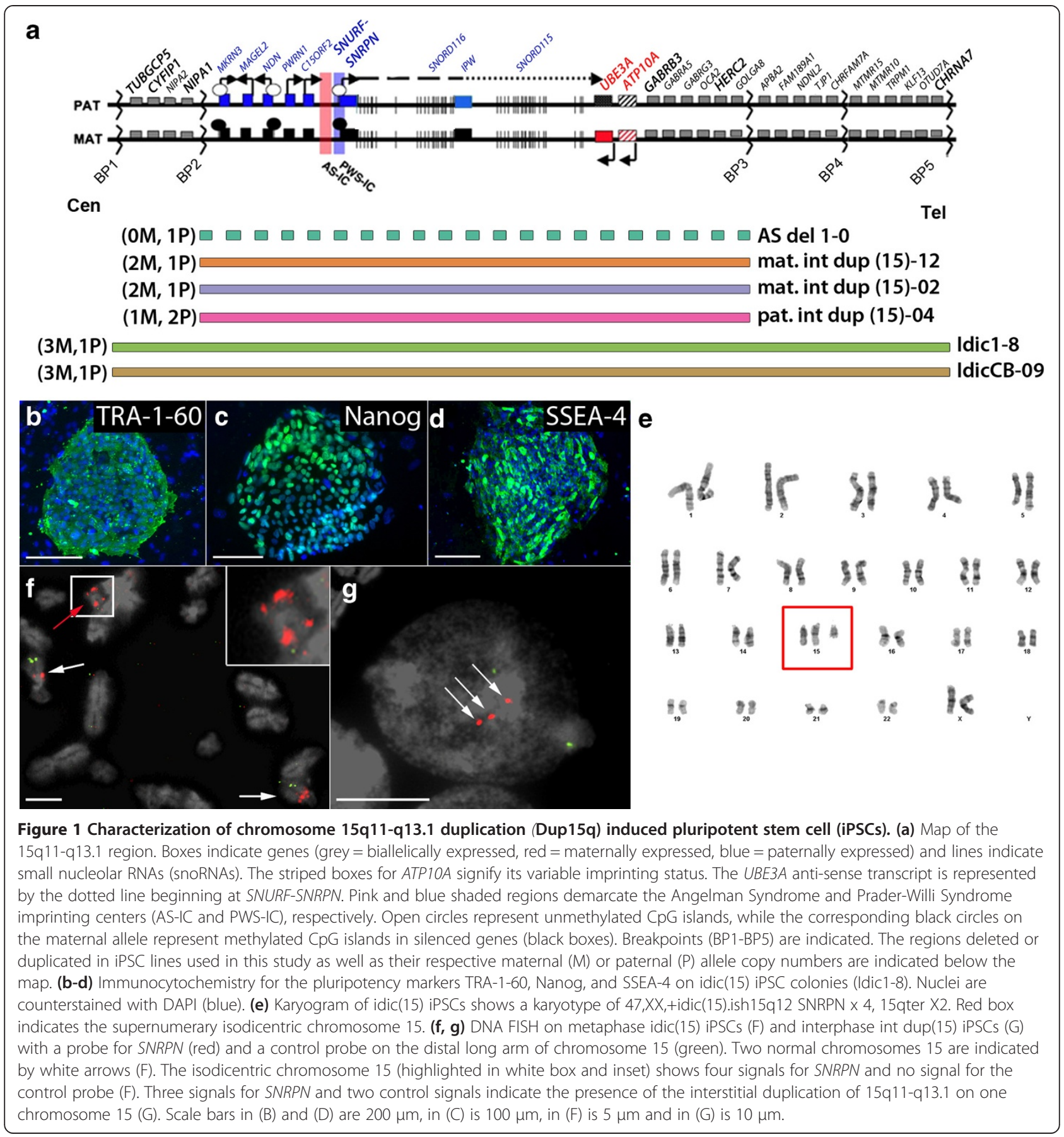

from $71 \%$ to $84 \%$ and paternal int dup(15) iPSCs had methylation levels ranging from $23 \%$ to $27 \%$ (Table 1 , Additional file 3: Table S2). Two iPSC lines from the mosaic int $\operatorname{dup}(15)$ individual had methylation levels of 54\% and 57\% [see Additional file 3: Table S2]. One line showed normal diploid cells by karyotype analysis (mat. int dup(15)-12), while the other was a mixed culture, having both duplication and normal cells (mat. int dup (15)-18). The remaining iPSC clones from the mosaic individual had methylation levels ranging from $65 \%$ to
68\% (Table 1). Thus, DNA methylation at the PWS-IC is consistent with the predicted copy number variations of the Dup15q cell lines and seems to be maintained during reprogramming.

To further investigate the maintenance of parental imprinting following reprogramming of patient cells into iPSCs and differentiation of iPSCs into neurons, we performed allele-specific RT-PCR for the paternally expressed imprinted in Prader-Willi syndrome (IPW) gene. We first identified a SNP (rs691) in the last exon of IPW and 


\begin{tabular}{ll} 
Table 1 Methylation analysis of chromosome \\
15q11-q13.1 duplication syndrome (Dup15q) induced \\
pluripotent stem cells (iPSCs) at the Prader-Willi \\
syndrome imprinting center (PWS-IC) \\
\hline Cell line & \% methylated at PWS-IC \\
\hline mat. Int dup(15) & $\mathbf{6 6 . 5 7} \pm \mathbf{0 . 9 2}$ \\
mat. Int dup(15)-02 & $66.15 \pm 2.12$ \\
mat. Int dup(15)-12 & $65.64 \pm 3.25$ \\
pat. Int dup(15) & $\mathbf{2 5 . 3 1} \pm \mathbf{1 . 4 6}$ \\
pat. Int dup(15)-04 & $23.53 \pm 2.93$ \\
Idic & $\mathbf{7 6 . 9 4} \pm \mathbf{5 . 0 2}$ \\
Idic1-8 & $72.43 \pm 1.00$ \\
IdicCB & $\mathbf{7 7 . 3 5} \pm \mathbf{3 . 5 2}$ \\
IdicCB-07 & $79.49 \pm 5.03$ \\
IdicCB-09 & $72.29 \pm 9.69$
\end{tabular}

${ }^{\mathrm{a} D N A}$ methylation at the PWS-IC was assayed by methylation specific qPCR using genomic DNA from Dup15q iPSCs. The average calculated percent methylation from all clones within a genotype is presented plus or minus the standard deviation in bold text. The averages calculated for percent methylation from three replicate assays each for selected clones plus or minus the standard

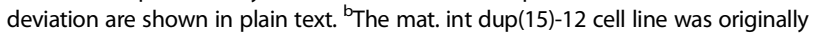
identified as an isogenic normal clone derived from the individual with maternal interstitial duplication. However, methylation analysis after approximately 25 subsequent passages suggests that this clone was mosaic and resolved to contain predominantly duplication containing cells. PWS-IC, Prader-Willi syndrome imprinting center; mat. Int dup(15), maternal interstitial duplication of 15q11-q13.1; pat. Int dup(15), paternal interstitial duplication of 15q11-q13.1; Idic, isodicentric chromosome 15; IdicCB, isodicentric chromosome 15 cord blood derived sample.

confirmed its heterozygosity in genomic DNA from the mat. int dup(15), pat. int $\operatorname{dup}(15)$, and idic(15) patient fibroblasts [see Additional file 4: Figure S2]. We were unable to investigate the SNP in genomic DNA from the umbilical cord blood idic(15) starting cell population; however, we did confirm heterozygosity in genomic DNA from the iPSCs derived from these cells. We then generated cDNA from the patient fibroblasts, iPSCs, and neural derivatives and, by sequencing across the SNP in $I P W$, confirmed that $I P W$ is expressed monoallelically and from the same allele as in the patient cell sample [see Additional file 4: Figure S2]. Together with the DNA methylation pattern at the PWS-IC, this data suggests that parental imprinting at the 15q11-q13.1 locus is maintained following reprogramming to iPSCs.

\section{Functional neurons derived from Dup15q iPSCs}

To prioritize individual iPSC lines based on their ability to differentiate into neurons, all clones of the Dup $15 \mathrm{q}$ iPSCs from each genotype were allowed to spontaneously differentiate in EB culture and were subjected to qRT-PCR using a custom PCR array containing a panel of neural genes and pluripotency genes [21]. Expression levels of all genes in the array were analyzed relative to those of EBs derived from AS iPSCs, which we previously reported to be capable of generating functional neurons [see Additional file 5: Figure S3A-D] [18]. Representative iPSC clones of each genotype were selected for future experiments based on low levels of expression of the pluripotency genes $N A N O G$ and zinc finger protein 42 (ZFP42) and expression levels of neural lineage genes, such as paired box 6 (PAX6), microtubule associated protein $2(M A P 2)$, and neural cell adhesion molecule 1 (NCAM1) that were similar to or higher than those of AS iPSC-derived EBs.

The selected iPSC clones from each Dup15q genotype were then differentiated using either an EB-based differentiation protocol that mimics embryonic development or a modified monolayer neural differentiation protocol $[19,20,31]$. Both protocols generated neural progenitors and subsequently post-mitotic neurons from all genotypes. Analysis of 10-week-old monolayer neuronal cultures by immunocytochemistry demonstrated the cultures largely consisted of MAP2-positive neurons (Figure 2a). Astrocytes, labeled by $\mathrm{S} 100$ calcium binding protein beta $(\mathrm{S} 100 \beta)$, were also observed (Figure $2 \mathrm{~b}$ ). The neuronal population contained both vesicular glutamate transporter 1 (VGlut1)-positive excitatory neurons and glutamate decarboxylate 65 (GAD65)-positive inhibitory neurons (Figure 2c and Figure 2d). Co-localization of the postsynaptic density protein PSD-95 and the presynaptic marker Synapsin I along MAP2-positive neurites indicates the formation of functional synapses in 10-week-old neuronal cultures (Figure 2e-e").

In order to compare neural cultures derived from the different cell lines used in this study, with respect to the types of cells present, we analyzed expression of neuronal (BIII-tubulin, RNA binding protein fox-1 homolog 3 (RBFOX3), and T-box brain 1 (TBR1)) and glial (S100 $\beta)$ genes in three independent neural cultures from each cell line. This analysis revealed that there was no significant difference in mean expression levels between cell lines [see Additional file 6: Figure S4A] (one-way ANOVA; $\beta I I I-$ tubulin: $\mathrm{F}_{6,14}=0.9295, P=0.5037 ;$ RBFOX3: $\mathrm{F}_{6,14}=1.938$, $P=0.1443 ;$ TBR1: $\mathrm{F}_{6,14}=2.174, P=0.1086 ;$ and $S 100 \beta$ : $\left.\mathrm{F}_{6,14}=2.786, P=0.0535\right)$. We also determined that all cell lines yielded neural cultures expressing similar levels of genes specific to excitatory (VGLUT2) and inhibitory (GAD1) neurons (Additional file 6: Figure S4A, one-way ANOVA; VGLUT2: $\mathrm{F}_{6,14}=2.597, P=0.0662$ and GAD1: $\left.\mathrm{F}_{6,14}=2.932, P=0.0456\right)$. Though the levels of expression varied between cell lines, we observed that all cell lines generated cells which expressed markers of dorsal forebrain including forkhead box G1 (FOXG1), PAX6, and orthdenticle homeobox 2 (OTX2) [see Additional file 6: Figure S4A] (one-way ANOVA; FOXG1: $\mathrm{F}_{6,14}=$ 11.75, $P<0.0001 ;$ PAX6: $\mathrm{F}_{6,14}=3.878, P=0.0172$; and OTX2: $\left.\mathrm{F}_{6,14}=11.97, P<0.0001\right)$. The midbrain specific gene engrailed 1 (EN1) was expressed in neural cultures from all cell lines but at noticeably lower levels 


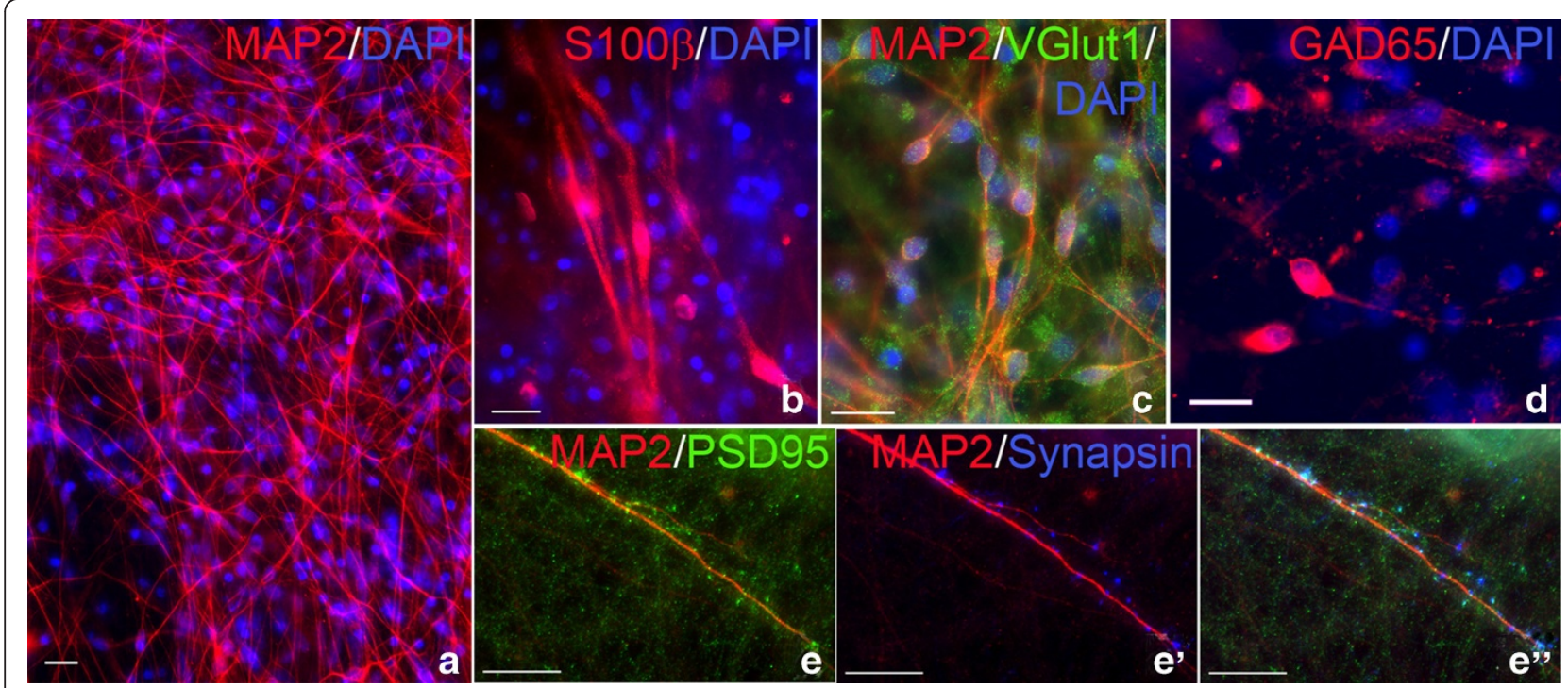

Figure 2 Characterization of neurons derived from chromosome 15q11-q13.1 duplication syndrome (Dup15q) induced pluripotent stem cells (iPSCs). (a) Immunocytochemistry for the neuronal marker MAP2 (red) indicates the presence of mature neurons in 10-week-old Idic1-8 iPSC-derived monolayer neuronal cultures. (b) S100ß-positive astrocytes (red) are also present after 10 weeks of neural differentiation. (c) VGlut1-positive (green) excitatory and (d) GAD-65-positive (red) inhibitory neurons show a mixed population of neurons. (e) MAP2-positive (red) neurons are decorated with PSD-95 (green) and (e') Synapsin (blue) puncta. (e") Merged image showing co-localization of PSD-95 and Synapsin suggests the formation of functional synapses. Nuclei are counterstained with DAPI (blue). All scale bars are $20 \mu \mathrm{m}$.

than the forebrain-specific genes [See Additional file 6: Figure S4A] (one-way ANOVA, $\mathrm{F}_{6,14}=7.664, P=0.0009$ ). We also assayed for expression of NK2 homeobox 2 (NKX2.1), a marker of ventral forebrain progenitors, and found that its levels were very low or undetectable in most cultures. Importantly, the replicate neural cultures used in these experiments included those derived by both monolayer culture and EB differentiation, demonstrating that there were no significant differences in the types of cells generated between the two protocols.

\section{Electrophysiological recordings of idic(15) iPSC-derived neurons}

We carried out electrophysiological recordings from differentiated neurons derived from the idic(15) iPSC line (Idic1-8) during in vitro development using the EB differentiation protocol. In 3-week-old cultures, most neurons ( $21 / 24$ cells) fired a single mature action potential in response to depolarizing current injection. In 6-weekold cultures, trains of action potentials were induced in more than half of the neurons recorded (29/51 cells). At weeks 12 to 14, a similar percentage of cells fired trains of mature action potentials and displayed large $(>1 \mathrm{nA})$ inward and outward currents in response to depolarization (Figure 3a-c). Inward sodium currents and action potentials were blocked by the sodium channel blocker tetrodotoxin ( $1 \mu \mathrm{M}$; data not shown). Spontaneous excitatory synaptic events were seen in about half of the neurons in 6-week-old cultures (28/52 cells), with an average frequency of $0.11 \pm 0.03 \mathrm{~Hz}(\mathrm{n}=28)$. In 14-week-old cultures, as shown in Figure 3d-e, isolated synaptic currents and occasional bursts of activity were seen in the majority of cells at a slightly higher frequency $(0.22 \pm 0.05 \mathrm{~Hz} ; \mathrm{n}=20)$. Spontaneous excitatory synaptic currents were blocked by the AMPA receptor antagonist 6-cyano-7-nitroquinoxaline-2,3-dione (CNQX) $(10 \mu \mathrm{M}$; data not shown). These data indicate that Dup15q neurons show in vitro maturation of intrinsic excitability and evidence of functional synapses, laying the groundwork for a detailed comparison of functional properties among different genotypes.

\section{Expression of 15q11-q13.1 genes in Dup15q iPSCs and iPSC-derived neurons}

The 15q11-q13.1 region includes several genes involved in neuronal structure and function (Figure 1a). Imprinting in the 15q11-q13.1 region results in allele-specific expression of several genes such that in non-neuronal cells (including pluripotent cells), SNRPN is expressed from the paternal allele and $U B E 3 A$ is expressed from both parental alleles. In neurons, however, $S N R P N$ is still expressed solely from the paternal allele, but $U B E 3 A$ becomes paternally silenced and is expressed only from the maternal allele [32]. While some studies suggest that $A T P 10 A$ is maternally expressed in the brain, others suggest that it is biallelically expressed or that its imprinting status varies among individuals [33]. Other genes in the region, including TUBGCP5, CYFIP1, NIPA1, GABRB3, 

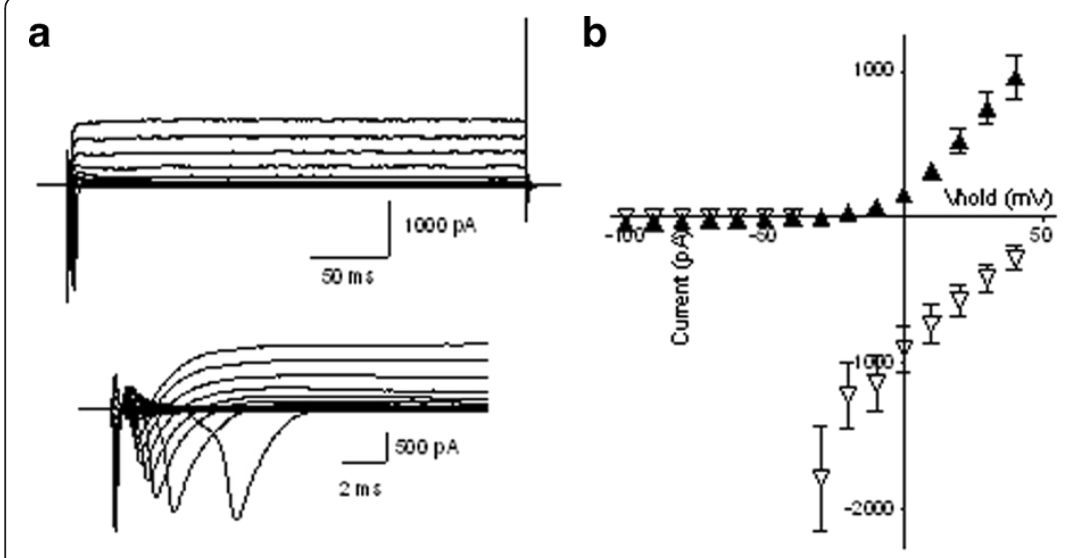

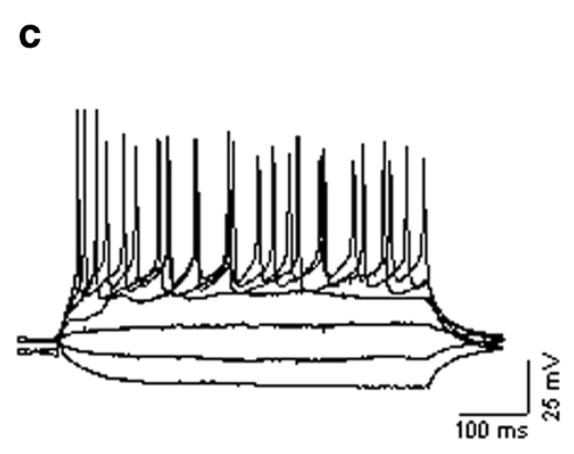

d

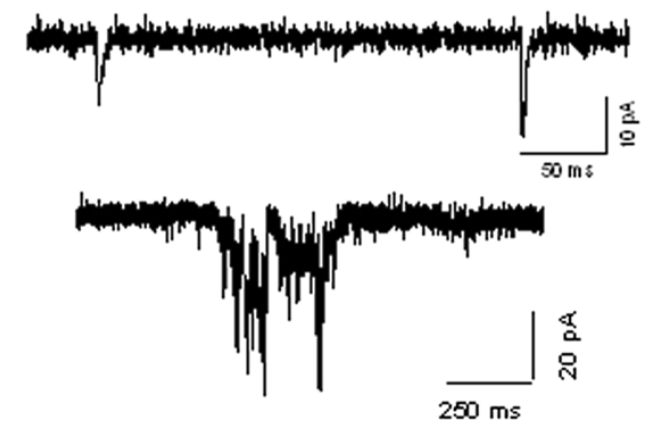

e

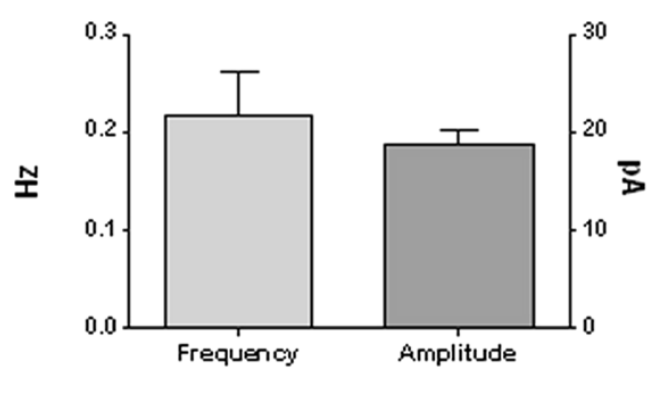

Figure 3 Electrophysiological characterization of induced pluripotent stem cell (iPSC)-derived neurons from a chromosome 15q11-q13.1 duplication syndrome (Dup15q) subject. (a) Representative whole cell potassium currents (top) and sodium currents (bottom) recorded from a 12-week-old Idic1-8 iPSC-derived neuron generated using the embryoid body based differentiation protocol. (b) Current-voltage relationship for peak sustained K current (filled triangles) and peak Na current (open triangles) from 12-week-old neurons ( $\mathrm{n}=16$ ). (c) Representative action potential train from a 12-week-old neuron. (d) Example sweeps of excitatory synaptic currents recorded from a 14-week-old neuron. (e) Group data showing average frequency and amplitude of synaptic currents in 14 week-old-neurons $(n=20)$.

HERC2, and CHRNA7 are expressed biallelically in all cell types tested to date. Evidence from analysis of both Dup15q lymphoblast and postmortem brain tissue samples suggests that expression levels of these genes differ significantly from normal individuals [34,35]. To investigate whether copy number variations of the 15q11-q13.1 region result in corresponding changes in the expression of genes located in the region, we performed qRT-PCR with iPSCs and iPSC-derived neurons (both monolayer and EB-derived) from all genotypes for TUBGCP5, CYFIP1, NIPA1, SNRPN, UBE3A, ATP10A, GABRB3, HERC2, and CHRNA7 [see Additional file 7: Figure S5A-B]. In each case, we compared the expression level of the gene in the disease-specific cells to the normal cells.

For all genotypes, expression of the 15q11-q13.1 genes in iPSCs generally corresponded to expressed copy number when compared to iPSCs derived from a control individual (Nml 1-0) (Figure 4a, top panel). The levels of expression of paternal-specific SNRPN in AS iPSCs and in the maternal int dup (15) and idic(15) iPSCs were similar to normal iPSCs because they only have a single active copy of the gene. The paternal int dup(15) iPSCs showed a twofold increase in SNRPN expression compared to normal iPSCs, reflecting the two active copies of the gene (Figure 4a, top panel; Additional file 7: Figure S5A). UBE3A, which is not imprinted in iPSCs was expressed at approximately half the level of normal in AS iPSCs and between two- and threefold of normal in idic(15) iPSCs. While UBE3A expression fell within the range of normal expression in both iPSC lines derived from the maternal int $\operatorname{dup}(15)$ sample and the paternal int $\operatorname{dup}(15)$ iPSCs, the average $U B E 3 A$ expression level in all three of these iPSC lines was consistently higher than that of normal iPSCs.

The expression levels of HERC2 and NIPA1 were not as predicted from copy number in some of the iPSC lines. HERC2 was expressed at levels near or slightly less than those seen in normal iPSCs in both the paternal and maternal int dup(15) iPSCs (Figure 4b, top panel; Additional file 7: Figure S5A). Expression of NIPA1 was roughly half that of normal iPSCs in both clones of the 


\section{a}
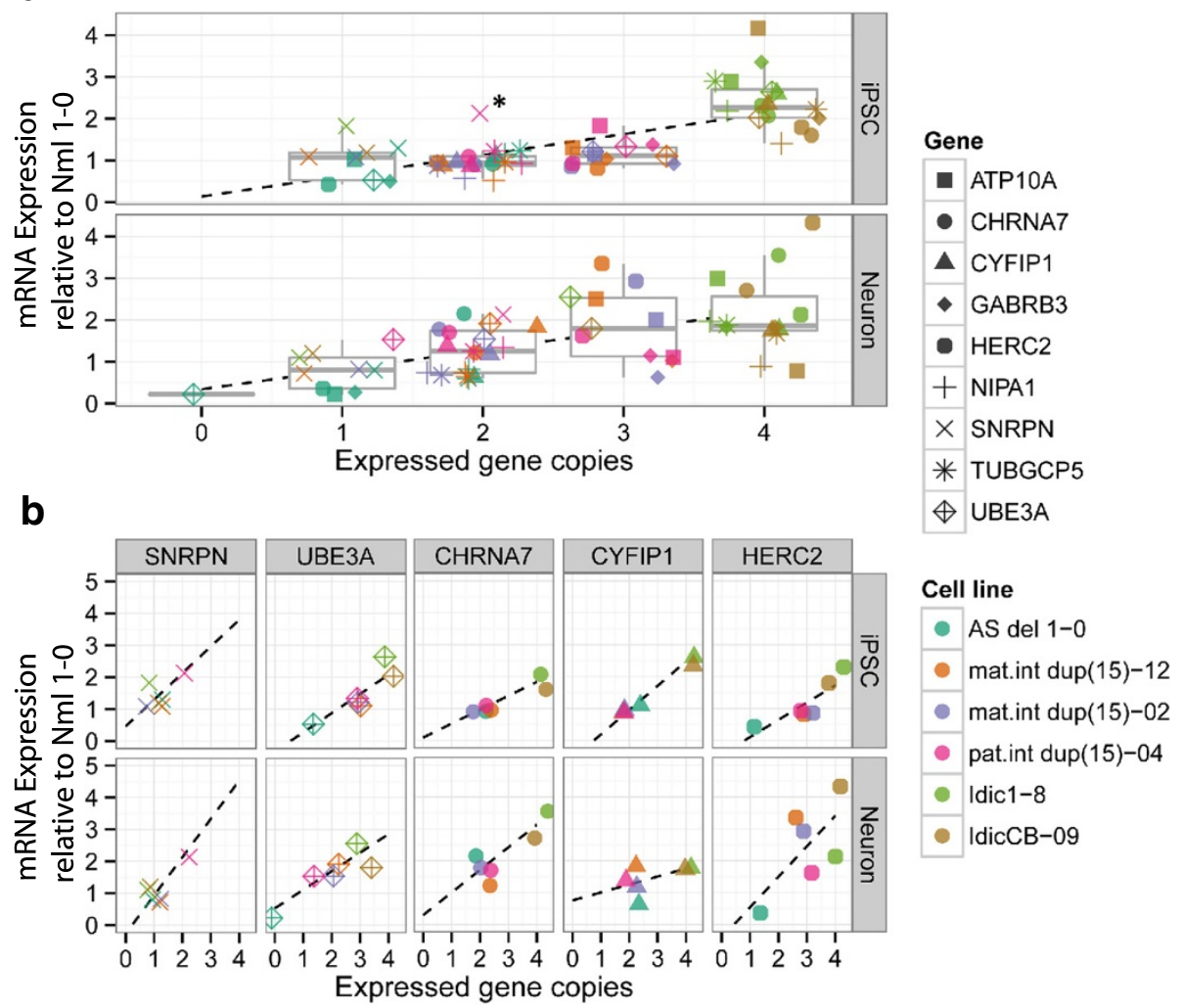

Figure 4 Expression of 15q11-q13.1 genes in chromosome 15q11-q13.1 duplication syndrome (Dup15q) induced pluripotent stem cells (iPSCs) and iPSC-derived neurons. (a) Box plots depicting the distribution of average mRNA expression levels of 15q11-q13.1 genes in Dup15q iPSCs (top) and 10-week-old iPSC-derived neurons (bottom) relative to normal samples ( $\mathrm{Nml} \mathrm{1-0)} \mathrm{as} \mathrm{they} \mathrm{correspond} \mathrm{to} \mathrm{the} \mathrm{number} \mathrm{of} \mathrm{expressed}$ copies of each gene. The dark gray line in the center of each box represents the median of the average relative mRNA expression levels of genes containing each number of expressed gene copies. Boxes indicate the 25th, 50th, and 75th percentiles. Whiskers represent the 5th and 95th percentiles. The data point for SNRPN in the pat. int dup(15)-04 iPSCs (marked by asterisk) is not indicative of unexpected mRNA expression levels relative to gene copy number, since this paternally expressed gene is duplicated on the paternal allele in this sample. (b) The average mRNA expression levels for selected 15q11-q13.1 genes in Dup15q iPSCs (top) and 10-week-old iPSC-derived neurons (bottom) relative to that of normal (Nml 1-0) iPSCs and neurons is presented in relation to the respective number of expressed copies of each gene.

maternal int dup(15) iPSC lines [see Additional file 7: Figure S5A].

Expression levels of most 15q11-q13.1 genes in 10week-old AS and idic(15) iPSC-derived neurons also largely correlated with their respective expressed copy numbers (Figure $4 \mathrm{a}$, bottom panel), with one notable exception. HERC2 expression in one of the idic(15) cell lines (IdicCB-09) was nearly 4.5-fold higher than normal, which is a deviation from the expected twofold increase due to these cells having two additional copies of the gene (Figure 4b, bottom panel, Additional file 7: Figure S5B). $U B E 3 A$ is imprinted in iPSC-derived neurons and, accordingly, $U B E 3 A$ expression levels were less than $25 \%$ of normal levels in AS iPSC-derived neurons. In idic (15) iPSC-derived neurons, UBE3A levels were about 2.5-fold higher than normal neurons, as expected for cells with three active copies of the gene (Figure 4b, bottom panel; Additional file 7: Figure S5B).
In the maternal int dup(15) iPSC-derived neurons (mat. int $\operatorname{dup}(15)-02$ ), we found that $U B E 3 A$ was expressed at the predicted 1.5-fold over normal. However, $U B E 3 A$ was also increased to a similar degree in the isogenic normal (mat. int dup(15)-12) neurons. In order to understand this apparent discrepancy in expected $U B E 3 A$ expression, we analyzed $U B E 3 A$ copy number in the maternal int $\operatorname{dup}(15)$ and isogenic normal clones using a qPCR CNV assay, and determined that both cell lines had three copies of $U B E 3 A$ [see Additional file 8: Figure S6A]. In addition, we detected RNA expression from three UBE3A alleles by RNAFISH with a $U B E 3 A$ BAC probe in iPSCs of both cell lines [see Additional file 8: Figure S6B]. This suggests that while initial analysis of iPSC clones, using DNA FISH for SNRPN and the PWS-IC methylation assay, indicated that the mat. int dup(15)-12 clone did not contain the 15q11-q13.1 duplication, this clone was 
also mosaic and over approximately 25 passages resolved to consist mostly of duplication-carrying cells. Therefore, we can interpret data generated using this cell line as a second maternal interstitial duplication clone.

15q11-q13.1 gene expression in the maternal int dup (15) and paternal int dup(15) iPSC-derived neurons did not correlate well with expressed copy number for several genes (Figure 4a, bottom panel). HERC2 levels were threefold higher than normal in maternal int $\operatorname{dup}(15)$ neurons, as opposed to the predicted 1.5-fold increase from the three expressed copies of the gene in these cells (Figure 4b, bottom panel). We also observed unexpected increases in expression of CYFIP1 and CHRNA7 in both the maternal and paternal int dup(15) iPSC-derived neurons (Figure 4b, bottom panel). We did observe the expected increase in SNRPN expression in the paternal int dup(15) neurons, however, expression of NIPA1, UBE3A, HERC2, and $C H R N A 7$ were also higher than expected based on expressed copy number.

Together, these data suggest that gene expression level correlates well with expressed copy number in iPSCs but less so in iPSC-derived neurons (compare top and bottom panels of Figure 4a). Furthermore, interstitial duplication iPSCs and neurons are more likely to have gene expression levels different from expected levels based on expressed copy number.

\section{Transcriptome analysis of AS, normal, and idic(15) iPSC-derived neurons}

We also sought to analyze gene expression genomewide. To test the hypothesis that alterations in gene expression would reflect either 1) disrupted pathways caused by altered dosage of the duplicated $15 q$ region or 2 ) the transcriptional responses to the physiological changes in the AS and idic(15) neurons, we performed strand-specific mRNA-Seq on 10-week old neurons generated from AS, normal, and idic(15) (Idic1-8) iPSCs. mRNA-Seq data were generated using neurons derived from two independent rounds of in vitro differentiation using the EB protocol from iPSCs of each of the three genotypes. Gene expression levels were compared between biological replicates and were found to be highly similar [see Additional file 9: Table S3, $\mathrm{r}^{2} \geq 0.97$ ]. The gene expression levels for the replicates were averaged and the AS and idic(15) samples were each compared to the normal sample.

Genes showing a twofold increase or decrease in expression compared to the normal sample were considered differentially expressed between those two samples. 5,369 genes were differentially expressed between idic(15) and normal iPSC-derived neurons (Figure 5a). A total of 1,667 genes were found to be differentially expressed between AS and normal iPSC-derived neurons (Figure 5a). Of these, 751 genes were differentially expressed in both samples compared to normal (Figure 5a). The number of genes found to be differentially expressed in both samples is greater than expected by chance (hypergeometric distribution with population size $\left.=27,297 ; P=1.88 \times 10^{-131}\right)$. Moreover, $76 \%$ of the genes differentially expressed in both AS and idic15 neurons show the same tendency (that is, both upregulated or both downregulated). Figure 5b shows a heat map of the top 200 most differentially expressed genes in both samples. Gene Ontology analysis was performed on the lists of genes specifically up- or downregulated in AS and idic(15) neurons compared to normal neurons [see Additional file 10: Table S4, Additional file 11: Table S5, Additional file 12: Table S6, and Additional file 13: Table S7]. The gene ontology term 'neuronal differentiation' was the most significantly enriched category from the lists of genes downregulated in both AS and idic(15) neurons $\left(P=9.7 \times 10^{-10}\right.$ and $P=4.31 \times 10^{-8}$ for AS and idic(15) neurons, respectively). Genes involved in cell cycle $\left(P=1.64 \times 10^{-23}\right)$ and protein catabolic processes $\left(P=5.96 \times 10^{-19}\right)$ were enriched among the list of genes upregulated in idic(15) neurons.

Interestingly, several known autism and epilepsy candidate genes, namely distal-less homebox 2 (DLX2), aristaless related homeobox $(A R X)$, ISL LIM homeobox 1 (ISL1), neuroligin 1 (NLGN1), SH3 and multiple ankyrin repeat domains 1 (SHANK1), adenosine A2A receptor (ADORA2A), and distal-less homeobox 5 (DLX5), were found in the list of genes differentially expressed and downregulated in AS or idic(15). To validate the transcriptome data and further interrogate some of these genes, qRT-PCR was performed on RNAs isolated from normal, AS and idic(15) (Idic1-8) neurons (Figure 6a). All seven of the genes assayed were shown to be downregulated in both AS and idic(15) iPSC-derived neurons. qRTPCR data from 19 different genes demonstrated agreement between qRT-PCR and mRNA-Seq data (Figure 6b). Although maternal int dup(15) iPSC-derived neurons were not included in the whole transcriptome analysis, we also assayed expression of the selected autism and epilepsy candidate genes in these neurons by qRT-PCR. We observed that, with the exception of $A D O R A 2 A$, expression of these genes was also downregulated in maternal int dup (15) iPSC-derived neurons when compared to normal neurons [see Additional file 14: Figure S7].

\section{Rescue of normal UBE3A expression levels in idic(15) iPSC-derived neurons}

To investigate whether the aberrant expression levels of $U B E 3 A$ in idic(15) neurons could be pharmacologically manipulated, we treated 10-week-old idic(15) iPSCderived monolayer neuron cultures with the DNA-binding compound mithramycin. This drug binds to GC-rich 


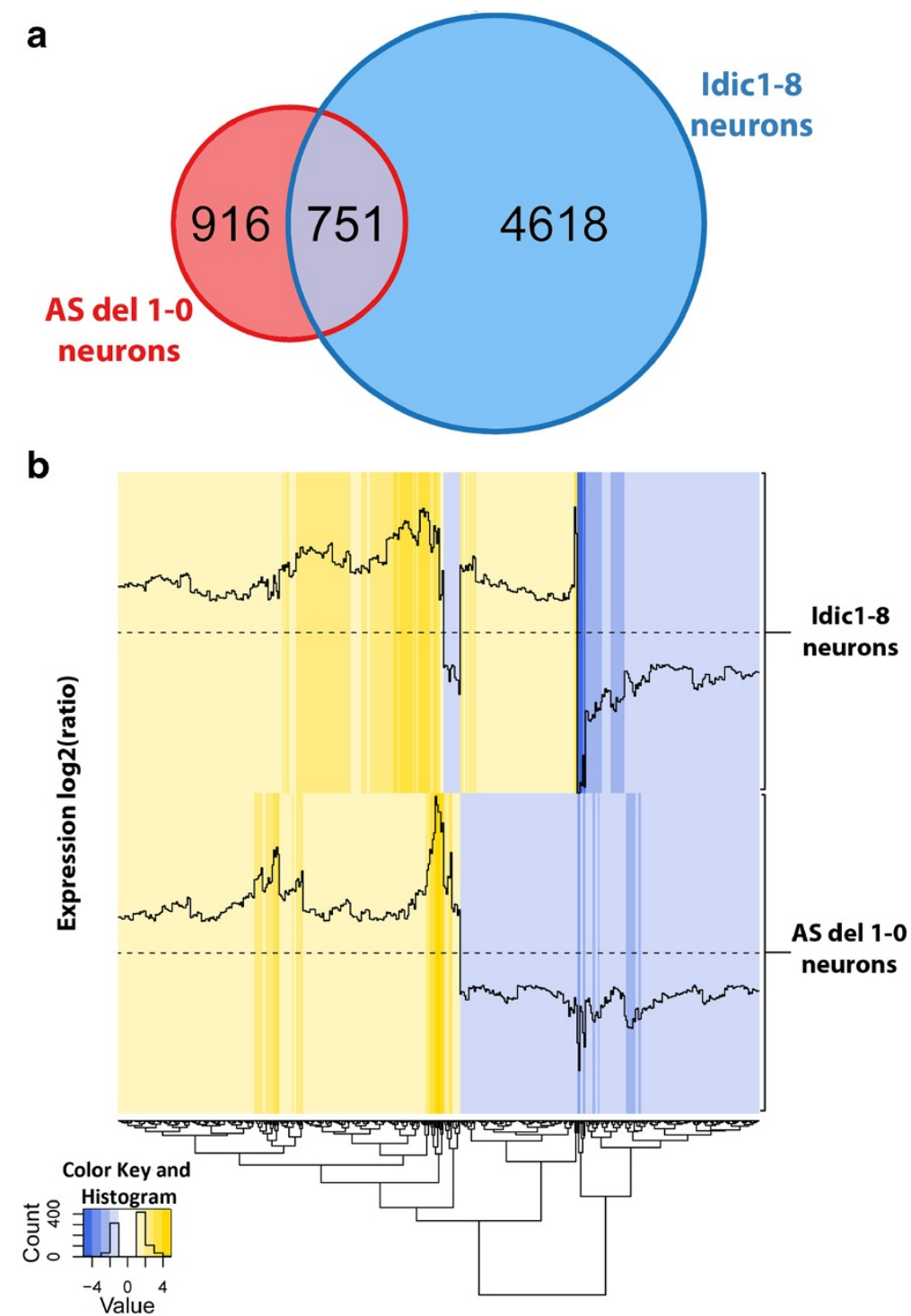

Figure 5 Most of the genes differentially expressed in both Angelman syndrome (AS) and isodicentric chromosome 15 (idic(15)) induced pluripotent stem cell (iPSC)-derived neurons compared to normal are changed in the same direction. (a) Venn diagram depicting the number of genes differentially expressed (twofold up or down) between AS (AS del 1-0) and idic(15) (Idic1-8) iPSC-derived neurons compared to normal (Nml 1-0) iPSC-derived neurons. The overlap of the two circles represents the set of shared differentially expressed genes in both AS and idic(15). (b) Heat map showing the mRNA expression levels relative to normal (Nml 1-0) of the top 200 genes differentially expressed in both AS (AS del 1-0) and idic(15)(Idic1-8) iPSC-derived neurons. Genes are clustered according to their similarity in gene expression levels across both samples. Genes colored in yellow show expression levels greater than normal, and genes colored in blue show expression levels less than normal. The trace lines indicate the expression levels of individual genes relative to normal. Normal expression level is indicated by the straight dotted lines.

sequences in specific gene promoters and competes with transcription factors that regulate gene expression $[36,37]$. We reasoned that since the $U B E 3 A$ promoter is $\mathrm{GC}$-rich and is known to be occupied by many transcription factors in various cell types [38], competition for binding by mithramycin may alter the levels of $U B E 3 A$ transcription. Following 72 hours of drug treatment, we found that $U B E 3 A$ mRNA levels in idic(15) neurons were reduced in a dose-dependent manner to levels near that of normal
iPSC-derived neurons (Figure 7a). These data suggest that $U B E 3 A$ can be pharmacologically regulated at the transcriptional level by mithramycin. However, competition for transcription factor binding by mithramycin is not specific for $U B E 3 A$ and occurs at many gene promoters. In line with this, we detected changes in expression levels of several other genes following mithramycin treatment, including the other 15q11-q13.1 genes SNRPN, GABRB3, and CYFIP1 [see Additional file 15: Figure S8]. 

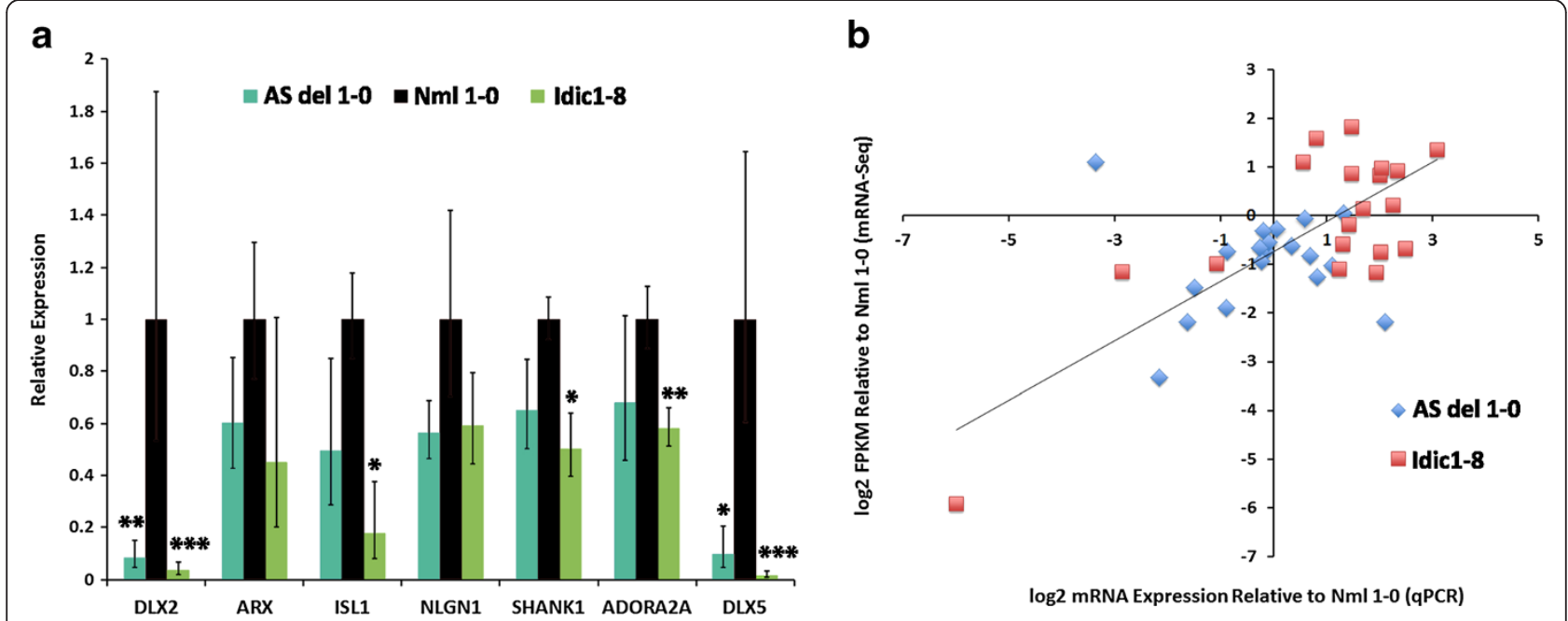

Figure 6 Quantitative reverse transcription PCR (qRT-PCR) validation of mRNA-Seq data. (a) Differential gene expression of select autism candidate genes and genes implicated in seizure disorders were verified by qRT-PCR in 10-week-old AS (AS del 1-0), normal (Nml 1-0), and idic(15) (Idic1-8) iPSC-derived neurons. $P$ values: ${ }^{*} P \leq 0.1,{ }^{* *} P \leq 0.05,{ }^{* * *} P \leq 0.01$. (b) Correlation of relative expression levels for 19 genes in iPSC-derived neurons as determined by mRNA-Seq and qRT-PCR. The log2-transformed RQ values for mRNA expression relative to normal neurons are plotted on the $x$-axis against the log2-transformed FPKM values obtained by mRNA-Seq analysis ( $y$-axis).

In an effort to identify possible mechanisms whereby mithramycin treatment rescues $U B E 3 A$ expression levels, we assayed transcription factor binding to $U B E 3 A$ by ChIP. It is well established that mithramycin inhibits binding of the Sp1 transcription factor and affects transcription of Sp1 target genes [37,39]. We were unable to detect Sp1 enrichment at several sites analyzed within the UBE3A promoter [see Additional file 16: Figure S9A]. For this reason, we instead analyzed binding of the transcription factor yin yang 1 (YY-1), which we confirmed does bind within the UBE3A promoter in both iPSCs and iPSC-derived neurons [see Additional file 16: Figure S9B]. YY-1 can act both as a transcriptional activator and repressor [40]. Transcriptional repression by YY-1 is thought to be mediated by recruitment of histone deacetylases and histone acetyltransferases [41]. We assayed binding of YY-1 to $U B E 3 A$ at two sites, one within exon 1 and one about 250 base pairs upstream of exon 1, following treatment of idic(15) iPSC-derived neurons for 72 hours with either $100 \mathrm{nM}$ mithramycin or DMSO. While there

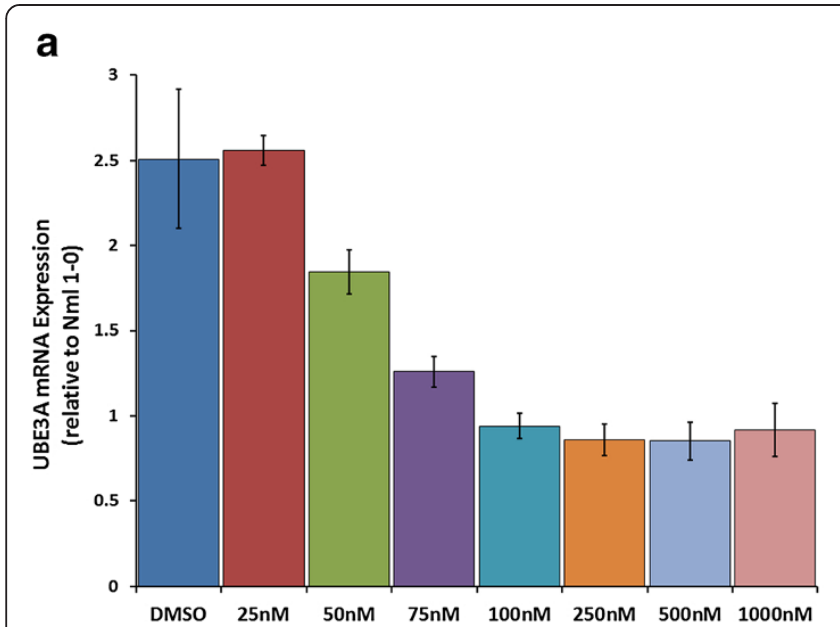

\section{b}

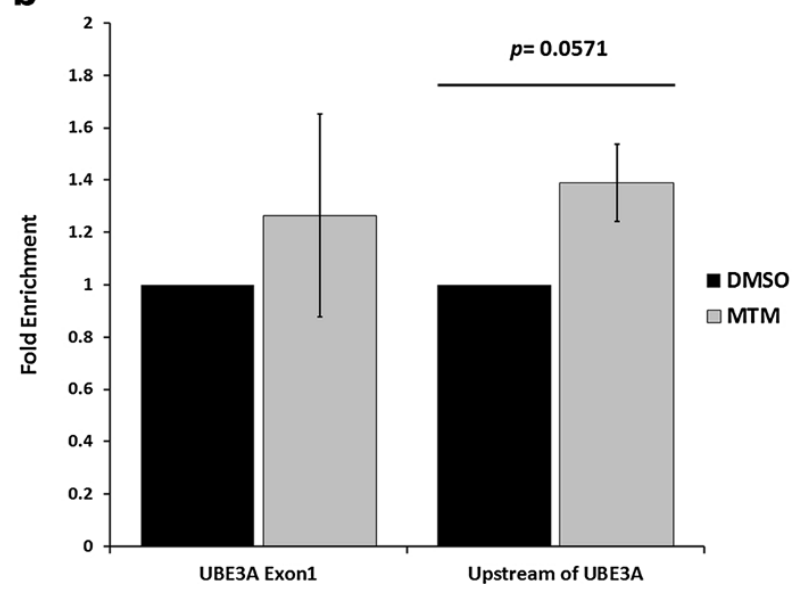

Figure 7 Rescue of normal UBE3A expression levels following treatment of isodicentric chromosome 15 (idic(15)) induced pluripotent stem cell (iPSC)-derived neurons with mithramycin. (a) qRT-PCR analysis of UBE3A mRNA expression in 10-week-old idic(15) (Idic1-8) iPSC-derived neurons following 72 hours of treatment with DMSO (vehicle) or mithramycin at concentrations ranging from $25 \mathrm{nM}$ to $1 \mu \mathrm{M}$. Data are presented as mean expression levels relative to that of untreated normal (Nml 1-0) iPSC-derived neurons. (b) ChIP-qPCR analysis of YY-1 binding at two sites in UBE3A, one within exon 1 and one upstream of exon 1, in 10-week-old idic(15) (Idic1-8) iPSC-derived neurons following 72 hours of treatment with either DMSO or 100 nM mithramycin. 
was no significant difference between the treatments in binding of YY-1 at exon 1, binding at the upstream site was increased in mithramycin-treated neurons to roughly 1.4fold that of DMSO-treated neurons (Figure $7 \mathrm{~b}, P=0.0571$ ). These data suggest that mithramycin treatment may recruit YY-1 to the $U B E 3 A$ promoter, where it can act in a repressive manner to decrease $U B E 3 A$ expression levels.

\section{Discussion}

Dup15q syndrome is a genetic form of autism in which the features of affected individuals closely resemble those in idiopathic autism $[1,2,8,9]$. In addition to the impairments in social interaction, verbal and nonverbal communication, and stereotyped behavior that underlie autism, individuals with Dup15q syndrome also suffer from hypotonia, seizures, developmental delay, and behavioral problems. We have generated iPSC models of Dup15q syndrome from a variety of patient samples containing different $\mathrm{CNVs}$ of the $15 \mathrm{q} 11-\mathrm{q} 13.1$ region. We show that the imprinting status of the PWS-IC is maintained following reprogramming of patient samples and that the ratio of methylated to unmethylated DNA at the PWS-IC confirms the expected 15q11-q13.1 copy number in each patient-derived iPSC line. Importantly, we demonstrated that all of the iPSC lines we generated can be differentiated into functionally mature neurons, as indicated by immunocytochemistry and electrophysiology. The ability to generate functional human neurons in large numbers from these iPSCs will enable us to perform molecular and cellular phenotypic analyses, and to test candidate therapeutic compounds in live human Dup15q neurons. Thus, these cell lines are an important resource for understanding Dup15q syndrome as well as idiopathic autism.

In contrast to AS, a single gene disorder which can result from loss of function of the $U B E 3 A$ gene alone, it is not known whether Dup15q syndrome results from overexpression of $U B E 3 A$ or from overexpression of $U B E 3 A$ plus one or more other genes in the 15q11-q13.1 region. Since maternal, but not paternal duplications typically lead to the Dup15q syndrome, a role for $U B E 3 A$ seems highly likely. However, the contribution of other genes has been difficult to explore since the only patient specimens available were blood or post-mortem tissues that may not accurately reflect the gene expression observed in live neurons. Our iPSC models of Dup15q allow for gene expression analysis in live human neurons carrying the various $15 \mathrm{q} 11-\mathrm{q} 13.1 \mathrm{CNVs}$. This experimental system also permits us to better resolve some of the confounding issues involved with post-mortem brain samples (variable mRNA or protein quality) and with genetically engineered transformed cell lines (unknown genetic and epigenetic aberrations) that have been previously used to study Dup15q syndrome $[34,42]$.
Our analysis of 15q11-q13.1 gene expression in undifferentiated iPSCs showed that, on the whole, expression levels in all genotypes correlated with 15q11-q13.1 copy number, including proper allele-specific expression of imprinted $S N R P N$. Consistent with previous findings in patient lymphoblasts [35], fibroblasts [43], and postmortem brain [34], we confirmed increased expression of $U B E 3 A$ in iPSCs derived from two separate idic(15) individuals. We also observed increased expression of several other genes included in the duplication region such as CYFIP1, GABRB3, and HERC2.

We found that following differentiation of iPSCs into functionally mature neurons, 15q11-q13.1 transcript levels did not consistently correlate with copy number. For example, CYFIP1 expression was increased relative to normal in both maternal and paternal int $\operatorname{dup}(15)$ neurons, which do not have a duplication of CYFIP1. Meanwhile, TUBGCP5 levels were significantly reduced in maternal int $\operatorname{dup}(15)$ neurons. In addition, a striking threefold increase in non-imprinted HERC2 expression was observed in maternal int $\operatorname{dup}(15)$ neurons, while the predicted expression level was 1.5-fold of normal. Interestingly, we also observed slight increases in expression of CHRNA7, a gene located well outside of the duplication region in both maternal and paternal int $\operatorname{dup}(15)$ neurons. AS iPSC-derived neurons, in which CHRNA7 copy number is unaffected, also showed a significant twofold increase of CHRNA7 compared to normal neurons. Together, these data suggest that, in neurons, transcription of genes both within and adjacent to the 15q11-q13.1 duplication region is not correlated to gene copy number. There are at least three possible reasons for these unexpected gene expression changes: 1) individual-to-individual variability; 2) disruption of long-distance regulatory elements by the chromosomal duplications; or 3) aberrant function of the neurons not associated with the chromosomal rearrangement.

Normal individual-to-individual variability is a confounding problem for gene expression studies in any human tissue type or patient-derived cell line. In some cases, the variability between individuals can be significant due to the different genetic backgrounds. We cannot rule out the possibility that the unexpected gene expression changes that we observe are due to normal variation. This appears unlikely, however, since the gene expression in the Dup15q iPSCs very closely reflects the copy number of the interrogated genes. The problems associated with normal variation can be addressed by examining samples from multiple individuals or by comparing isogenic Dup15q and normal cell lines. We have compared gene expression in iPSC-derived neurons from two different idic(15) individuals, and they show similar expression levels of all genes, except for ATP10A and $H E R C 2$. ATP $10 A$ is highly variable between replicate 
neurons from the same individual and has proven difficult to analyze even in mouse models where the genetic background is the same [44]; thus, changes in its expression levels are difficult to interpret. HERC2 encodes a ubiquitin ligase that has been shown to act in a complex with UBE3A [45]. The increased HERC2 levels could conceivably influence the severity of the symptoms in some idic(15) individuals. Isogenic cell lines can be obtained by deriving Dup15q and normal cell lines from the same mosaic individual, as attempted for our maternal interstitial duplication individual, or by genetically correcting the duplication in individual cell lines. These comparisons will be important future studies for Dup15q syndrome.

Our results indicate that the correlation between gene expression level and copy number is different in interstitial versus isodicentric 15q11-13.1 duplications, and we suspect that alterations in long range chromatin regulation may be involved. Several models have been proposed to explain how deletions and duplications of genes or large regions of DNA can alter transcription of a given gene. In particular, regulatory sequences can be disrupted by direct mutation of promoters and enhancers. Alternately, binding sites for proteins that mediate the necessary chromatin looping required for proper gene expression can be disrupted. Abrogation of long-distance transcriptional regulation due to chromosomal rearrangements has been implicated in several human diseases [46], and our data may suggest such a mechanism in Dup15q syndrome. We hypothesize that chromatin-organizing regulatory elements exist near the duplication/deletion breakpoints. The function of these regulatory elements may be disrupted by the duplication or deletion events. The effect of this regulatory disruption is predicted to affect interstitial duplications and deletions more than idic(15) samples, consistent with our results.

It is possible, however, in neurons that undergo silencing of the paternal $U B E 3 A$ allele and complex changes in chromatin structure that transcription from the extra maternal $U B E 3 A$ alleles is subject to differential regulation [47]. Studies using an engineered neuroblastoma cell line model of Dup15q showed aberrant expression levels of several 15q11-q13.1 genes compared to levels predicted by copy number [42]. Meguro-Horike et al. propose that the discordance in transcript levels compared to copy number of 15q11-q13.1 genes is a result of a disruption of transcriptional regulation normally mediated either by pairing of homologous chromosomes or by intra-chromosomal pairing in cells containing extra 15q11-q13.1 alleles [42].

We also investigated genome-wide gene expression differences between idic(15), AS, and normal iPSC-derived neurons. Altered $U B E 3 A$ gene dosage is proposed to underlie most of the phenotypic consequences of Dup15q syndrome $[4,8,14]$. We reasoned that gene expression changes that are caused by increases in $U B E 3 A$ expression in Dup15q neurons should be reversed in AS neurons that have decreased $U B E 3 A$ expression. In addition to its role in ubiquitination, $U B E 3 A$ acts as a transcriptional co-activator. UBE3A can also affect gene transcription via ubiquitination of protein targets in signaling pathways or by directly changing expression levels of individual genes. In both cases, the $U B E 3 A$-mediated effects on transcription should be opposite between idic(15) and AS neurons. As expected, there were more changes between idic(15) and normal than AS and normal neurons. This is likely due to the fact that the idic(15) neurons have two extra copies of a $9.6 \mathrm{Mb}$ region, while AS neurons have one less copy of a $5.1 \mathrm{Mb}$ region.

When considering the genes differentially expressed in both AS and idic(15) neurons compared to normal neurons, we found more similarities than differences. A remarkable $75 \%$ of all genes found differentially expressed and common to both idic(15) and AS neuron samples showed a similar expression pattern (that is, both downregulated or both upregulated), contrary to our hypothesis that AS and idic(15) are 'opposite' disorders. In fact, the same gene ontology term was identified amongst genes downregulated in both AS and idic(15) neurons. Since these gene expression changes represent changes at the mRNA level and not necessarily the protein level, we speculate that the shared changes result from a common neuronal response to malfunctioning synapses. We suspect that these gene expression changes are secondary to the chromosomal duplication or deletion, and may even occur in neurodevelopmental disorders not involving the chromosome 15q11-q13.1 region. Consistent with this idea, the M12 gene module identified by Voineagu et al. [48], that is transcriptionally downregulated in postmortem brain samples from idiopathic autism cases shares similar gene ontology terms to those downregulated in idic(15) and AS neurons, including terms involving synapses, axons, and neuron projections.

The major categories of genes upregulated in idic(15) neurons include genes involved in the cell cycle and those involved in protein catabolism, including those involved in ubiquitin-mediated proteolysis. Why the cell cycle would be affected in postmitotic neurons is perplexing. Growing evidence suggests that abnormal expression of cell cycle genes can participate in neuronal death, but neither increased nor decreased neuronal death has been observed in Dup15q syndrome or in our cell culture models. A recent report showed that the retinoblastoma 1 (RB1) protein is required for continuous cell cycle repression and neuronal survival [49], and it is becoming more apparent that post-mitotic neurons actively repress the cell cycle [50]. It is possible that the upregulated cell cycle genes serve to repress the cell cycle and may represent more rapid maturation of the idic(15) in vitro differentiated 
neurons. Indeed, $R B 1$ is one of the upregulated cell cycle genes. There are also several proteasome subunit and ubiquitin-mediated proteolysis genes that are included among the cell cycle genes, thereby contributing to the significance of this gene ontology term. The upregulation of genes involved in protein catabolism and ubiquitin mediated proteolysis is somewhat expected, although it is interesting that a large number of genes involved in this pathway are upregulated. Ube3a was recently shown to regulate protein homeostasis by directly ubiquitinating Rpn10, a proteasomal shuttling protein, in Drosophila melanogaster [51]. If Rpn10 is shown to be a target of UBE3A in human neurons, this could explain the dysregulation of protein catabolism in idic(15) neurons.

We sought to reduce $U B E 3 A$ mRNA levels in idic(15) neurons. Using our iPSC model of idic(15), we were able to perform a proof-of-principle experiment to determine whether $U B E 3 A$ levels could be pharmacologically reduced in human neurons. We found that treatment of idic(15) iPSC-derived neurons with the DNA binding compound mithramycin, an anti-tumor antibiotic, was able to reduce $U B E 3 A$ mRNA to levels similar to that of normal neurons. While several studies have shown that mithramycin inhibits transcription by competing for binding at GC-rich regions with the transcription factor Sp1 [37,39], we were unable to demonstrate Sp1 binding at $U B E 3 A$ by ChIP in our neurons after trying three different anti-Sp1 antibodies. We did, however, make the novel observation that binding of YY-1 is increased following mithramycin treatment. Since YY-1 can act as both a transcriptional activator and repressor [40], we hypothesize that YY-1 acts in a repressive manner at $U B E 3 A$ as increased binding is associated with a reduction in $U B E 3 A$ transcript following mithramycin treatment. YY-1 mediated transcriptional repression has been shown to involve recruitment of histone deacetylases and histone acetyltransferases and thereby establishment of repressive chromatin modifications [41]. While mithramycin is an FDA-approved drug used in the treatment of various cancers [52-54], we do not suggest that it is suitable for use in the clinical treatment of Dup15q, due to its effects on the expression of many other genes. Our findings do demonstrate the feasibility of using Dup15q iPSC-derived neurons to screen for other therapeutic compounds with the aim of restoring normal $U B E 3 A$ expression levels.

\section{Conclusions}

We have derived iPSC lines from two idic(15) individuals, one maternal int dup(15) individual and one paternal int dup(15) individual. These iPSCs can be differentiated into electrophysiologically mature neurons. These cell lines are an important resource for studies of Dup15q syndrome and idiopathic autism. Our analysis of gene expression in iPSCs and iPSC-derived neuronal cultures containing varied CNVs of the 15q11-q13.1 region supports the theory that altered expression of several genes in this region may underlie the Dup15q phenotype. While altered $U B E 3 A$ gene dosage is often proposed to be the mechanism responsible for Dup15q syndrome, our data along with data from other studies $[8,34,42]$ suggest that disruption of additional candidate genes may contribute significantly. Altered expression of such additional genes may be due to either their presence in the duplication region or to disruption of their transcriptional regulation as a result of the chromosomal rearrangement (and thus independent of copy number). As a result, our data also point to possible disruption of transcriptional regulation of genes in the duplication region in iPSC-derived neurons. Finally, we restored $U B E 3 A$ mRNA levels in idic(15) neurons to normal levels using the antitumor antibiotic, mithramycin, demonstrating that UBE3A mRNA levels could be manipulated pharmacologically. Future studies in which the expression levels of individual genes are reduced or that compare Dup15q neurons with isogenic control neurons may help delineate the precise roles of individual genes in this disorder.

\section{Availability of supporting data}

The data set supporting the results of this article is available in the National Center for Biotechnology Information (NCBI) Sequence Read Archive (SRA), under the accession number SRP044749 (http://www.ncbi.nlm. nih.gov/sra/?term=srp044749).

\section{Additional files}

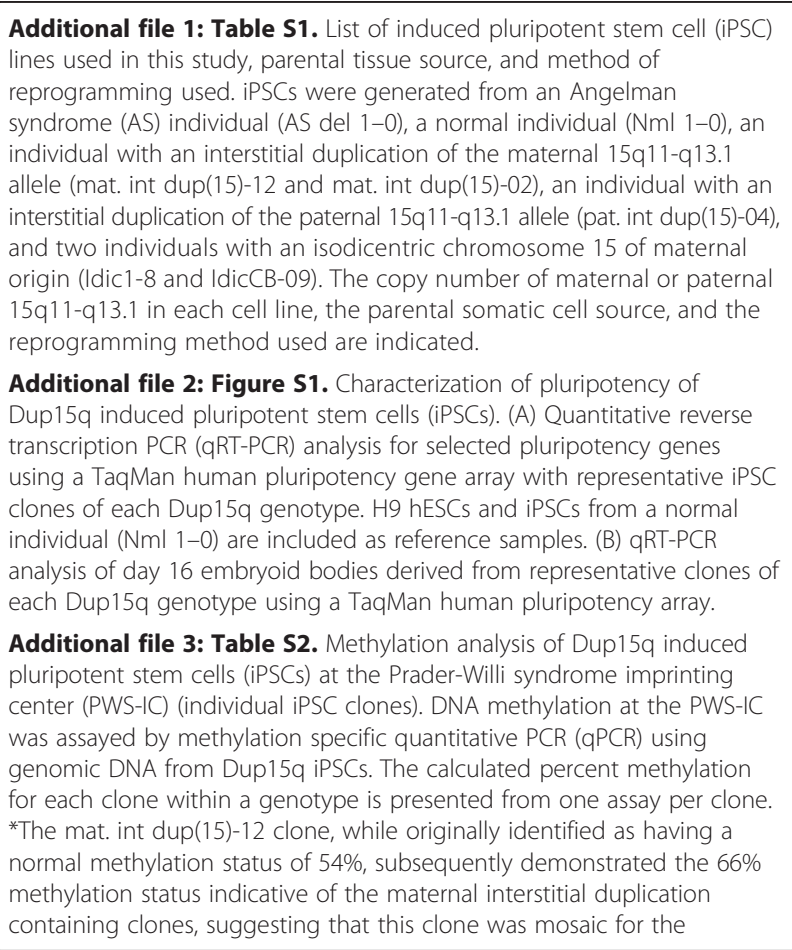


duplication. ** The mat. int dup(15)-18 clone was also shown to be a mixed clone by DNA fluorescence in situ hybridization (FISH) analysis using a probe for SNRPN.

Additional file 4: Figure S2. Allele-specific reverse transcription PCR (RT-PCR) for paternally expressed imprinted in Prader-Willi syndrome (IPW) in patient fibroblasts, induced pluripotent stem cells (iPSCs), and iPSC-derived neurons. DNA sequencing of genomic DNA from either patient fibroblasts (mat. int dup(15), pat. int dup(15), and idic(15)) or iPSCs (idic(15) cord blood (IdicCB-09)) indicates the presence of a polymorphic single nucleotide polymorphism (SNP), rs691, in the last exon of the imprinted in Prader-Willi syndrome (IPW) gene (arrows). Sequencing of CDNA derived from patient fibroblasts, iPSCs, and 10-week-old iPSC-derived neurons demonstrates that expression of IPW in all samples is monoallelic. Expression of the same allele (as indicated by DNA sequencing traces containing the same nucleotide at rs691, (asterisks)) in fibroblasts, iPSCs, and iPSC-derived neurons suggests that the parental imprinting of IPW is maintained following reprogramming and differentiation of iPSCs into neural derivatives. We were unable to analyze cDNA from pat. int dup(15) fibroblasts and idic(15) cord blood due to a lack of sufficient patient cells for generating RNA.

Additional file 5: Figure S3. Analysis of neural differentiation of Dup15q induced pluripotent stem cell (iPSC) clones using a custom neural differentiation quantitative PCR (qPCR) array. Each iPSC clone derived from Dup15q patient samples was assayed for neural differentiation capacity using a custom TaqMan neural differentiation QPCR array. iPSCs derived from individuals with a maternal interstitial duplication (A), paternal interstitial duplication (B), or idic(15) (fibroblast sample, C, and umbilical cord blood, D) were spontaneously differentiated via embryoid body formation for 16 days. Neural gene expression levels were analyzed relative to expression in AS del 1-0 day 16 embryoid bodies. AS del 1-0 is used as a calibrator sample since it has previously been published to readily generate mature neurons and astrocytes [18]. Embryoid bodies derived from H9 hESCs and normal iPSCs ( $\mathrm{Nml} 1-0)$ were included in analysis for comparison purposes. The pluripotency genes NANOG and ZFP42 were also included in analysis to assay downregulation of pluripotency genes during differentiation.

Additional file 6: Figure S4. Characterization of Dup15q induced pluripotent stem cell (iPSC)-derived neural cultures. (A) Triplicate independently-derived 10-week-old neural cultures from each Dup15q cell line were analyzed by qRT-PCR for markers of neurons and glia (BIII-tubulin, RBFOX3, TBR1, and S1O0B) excitatory and inhibitory neurons (VGLUT2 and GAD1), and forebrain and midbrain neurons (FOXG1, PAX6, OTX2, and EN1). Data are presented as mean expression relative to GAPDH. (B) Mean expression levels of each gene were compared between cell lines using one-way ANOVA followed by Tukey's multiple comparison post hoc test. Pairwise comparisons having significant $P$ values $(P<0.05)$ for each gene are listed.

Additional file 7: Figure S5. Expression of 15q11-q13.1 genes in Dup15q induced pluripotent stem cell (PSCs) and iPSC-derived neurons. (A) qRT-PCR analysis of selected 15q11-q13.1 genes in iPSCs. iPSC lines are presented from left to right in order of increasing 15q11-q13.1 copy number. Genes are arranged along the $x$-axis in the order of their location within the 15q11-q13.1 region. (B) qRT-PCR analysis of selected 15q11-q13.1 genes in 10-week-old iPSC-derived neurons.

Additional file 8: Figure S6. Analysis of UBE3A copy number in induced pluripotent stem cell (iPSCs). (A) The genomic copy number of UBE3A was analyzed by qPCR in each iPSC line using TaqMan Copy Number Assays. UBE3A copy number was calculated using RNase $P$ as an endogenous reference. Error bars indicate standard error of the mean. (B) RNA FISH for UBE3A in IPSCS shows RNA expression from two (Nml 1-0), three (mat. int dup(15)-02 and mat. int dup(15)-12) or four (Idic1-8) UBE3A alleles. Arrows indicate positive FISH signal. Nuclei are labeled with DAPI (blue).

Additional file 9: Table S3. mRNA-Seq data from AS (AS del1-0), normal (Nml 1-0), and idic(15) (Idic1-8) induced pluripotent stem cell (iPSC)-derived neurons. The tab titled "transcriptome data in neurons" shows the averaged fragments per kilobase pair per million mapped reads (FPKM) values for mRNA-Seq performed on two biological replicates of AS del1-0, Nml1-0, and Idic1-8 iPSC-derived neurons. The "AS vs. Normal" tab shows a scatter plot comparing the Log2 transformed FPKM+1 values for AS del1-0 and Nml1-0 iPSC-derived neurons. The "Idic(15) vs. AS" tab shows a scatter plot comparing the Log2 transformed FPKM+1 values for Idic1-8 and AS del1-0 iPSC-derived neurons. The "Idic (15) vs. Normal" tab shows a scatter plot comparing the Log2 transformed FPKM+1 values for Idic1-8 and Nml1-0 iPSC-derived neurons. The "Idic(15) vs. Idic(15)" tab shows a scatter plot comparing the Log2 transformed FPKM+1 values for the two biological replicates of Idic1-8 iPSC-derived neurons.

Additional file 10: Table S4. Gene Ontology analysis of genes upregulated in idic(15) (Idic1-8) induced pluripotent stem cell (iPSC)-derived neurons. Genes upregulated in idic(15) iPSC-derived neurons at least twofold compared to normal were subject to analysis using DAVID (http://david.abcc.ncifcrf.gov). Gene ontology terms representing the function of the genes upregulated are shown along with the corresponding statistical analysis.

Additional file 11: Table S5. Gene Ontology analysis of genes upregulated in AS del 1-0 induced pluripotent stem cell (iPSC)-derived neurons. Genes upregulated in AS iPSC-derived neurons at least twofold compared to normal were subject to analysis using DAVID (http://david.abcc. ncifcrf.gov). Gene ontology terms representing the function of the genes upregulated are shown along with the corresponding statistical analysis.

Additional file 12: Table S6. Gene Ontology analysis of genes downregulated in idic(15) (Idic1-8) induced pluripotent stem cell (iPSC)-derived neurons. Genes downregulated in idic(15) iPSC-derived neurons at least twofold compared to normal were subject to analysis using DAVID (http://david.abcc.ncifcrf.gov). Gene ontology terms representing the function of the genes downregulated are shown along with the corresponding statistical analysis.

Additional file 13: Table S7. Gene Ontology analysis of genes downregulated in AS del 1-0 induced pluripotent stem cell (iPSC)-derived neurons. Genes downregulated in AS iPSC-derived neurons at least twofold compared to normal were subject to analysis using DAVID (http://david.abcc.ncifcrf.gov). Gene ontology terms representing the function of the genes downregulated are shown along with the corresponding statistical analysis.

Additional file 14: Figure S7. Analysis of autism candidate genes in maternal int dup(15) induced pluripotent stem cell (iPSC)-derived neurons. Differential gene expression of select autism candidate genes and genes implicated in seizure disorders were analyzed by qRT-PCR in 10-week-old normal (Nml 1-0), maternal int dup(15) (mat int dup(15)-02), and idic(15) (Idic1-8) iPSC-derived neurons. $P$ values: ${ }^{*} P \leq 0.1,{ }^{* *} P \leq 0.05$, ${ }^{* * *} P \leq 0.01$.

Additional file 15: Figure S8. Off-target effects of mithramycin treatment in idic(15) induced pluripotent stem cell (iPSC)-derived neurons. qRT-PCR analysis of SNRPN, GABRB3, and CYFIP1 expression in 10-week-old idic(15) (Idic1-8) iPSC-derived neurons following 72 hours of treatment with mithramycin or DMSO. $P$ values: ${ }^{*} P \leq 0.1,{ }^{* *} P \leq 0.05,{ }^{* * *} P \leq 0.01$.

Additional file 16: Figure S9. Chromatin immunoprecipitation (ChIP) analysis of Sp1 and YY-1 binding at the UBE3A promoter. (A) ChIP-qPCR assays were performed on idic(15) (Idic1-8) iPSC-derived neurons using two different antibodies against Sp1. With anti-Sp1 antibody \#1 (Santa Cruz Biotechnology, Inc.), we were unable to detect significant enrichment over background signal (lgG) suggesting that this antibody does not work in our hands. Using anti-Sp1 antibody \#2 (Cell Signaling Technologies), we detected low levels of enrichment at two sites at UBE3A (one within exon 1 and one upstream of exon 1), however, when compared to enrichment at the Sp1 promoter and the DHFR promoter two positive control locations, we concluded that binding of Sp1 to UBE3A is not significant. (B) ChIP-qPCR assays were performed on idic(15) (Idic1-8) iPSC-derived neurons to analyze binding of YY-1 to two locations at $U B E 3 A$, one within exon 1 and one upstream of exon 1. Data are presented as the mean enrichment from triplicate ChIP experiments plus or minus the standard error of the mean.

\section{Abbreviations}

ADORA2A: adenosine A2A receptor; ARX: aristaless related homebox: AS: Angelman syndrome; ASD: autism spectrum disorder; ATP10A: ATPase 
class V 10A; cAMP: cyclic adenosine monophosphate; ChIP: chromatin immunoprecipitation; CHRNA7: cholinergic receptor nicotinic alpha 7 ; CNQX: 6-cyano-7-nitroquinoxaline-2,3-dione; CNV: copy number variation; CYFIP1: cytoplasmic FMRP interacting protein; DLX2: distal-less homebox 2; DLX5: distal-less homeobox 5; DMSO: dimethyl sulfoxide;

Dup15q: chromosome 15q11-q13.1 duplication; EB: embryoid body; EN1: engrailed 1; FISH: fluorescence in situ hybridization; FMRP: fragile $X$ mental retardation protein; FOXG1: forkhead box G1; FPKM: fragments per kilobase gene model per million base pairs; GABA: gamma-aminobutyric acid; GAD65: glutamate decarboxylase; GAPDH: glyceraldehyde-3-phosphate dehydrogenase; HERC2: HECT and RLD domain contained E3 ubiquitin protein ligase 2; idic(15): isodicentric chromosome 15; int dup(15): interstitial duplication of 15q11-q13.1; iPSC: induced pluripotent stem cell; IPW: imprinted in Prader-Willi syndrome; ISL1: ISL LIM homeobox 1; KLF4: Kruppel-like factor 4; LIN28: lin28 homolog A; MAP2: microtubule associated protein 2; MEF: mouse embryonic fibroblasts; MYC: v-myc avian myelocytomatosis viral oncogene homolog; NCAM1: neural cell adhesion molecule 1; NCBI: National Center for Biotechnology Information; NIPA1/ NIPA2: nonimprinted in Prader-Willi/Angelman syndrome region 1 and 2; NKX2.1: NK homeobox 2; NLGN1: neuroligin 1; OCT4: POU class 5 homeobox 1; OTX2: orthodenticle homeobox 2; PAX6: paired box 6; PSD95: postsynaptic density protein 95; PWS-IC: Prader-Willi syndrome imprinting center; qRT-PCR: quantitative reverse transcription polymerase chain reaction; RBFOX3: RNA binding protein, fox-1 homolog 3 (NEUN); RB1: retinoblastoma 1; SHANK1: SH3 and multiple ankyrin repeat domains 1; SNP: single nucleotide polymorphism; SNRPN: small nuclear ribonucleoprotein N; SOX2: SRY-box 2; SSEA4: state specific embryonic antigen 4; S100ß: S100 calcium binding protein beta; TBR1: T-box brain 1; TUBGCP5: tubulin gamma complex associated protein 5; UBE3A: ubiquitin protein ligase E3A; VGlut1: vesicular glutamate transporter 1; YY-1: yin yang 1; ZFP42: zinc finger protein 42.

\section{Competing interests}

All authors declare that they have no competing interests.

\section{Authors' contributions}

NDG and SJC conceived of and designed the study, collected and analyzed data, and wrote the manuscript. P-FC, JJF, and TMR collected and analyzed data. AMP analyzed data and provided critical revision of the manuscript. HG-D prepared sequencing libraries. JB collected and analyzed data and provided critical revision of the manuscript. ESL designed study, analyzed data, and wrote the manuscript. LTR acquired and processed patient samples and provided critical revision of the manuscript. BRG designed the study, analyzed data, and provided critical revision of the manuscript. ML conceived of and designed the study and provided critical revision of the manuscript. All authors read and approved the final manuscript.

\section{Acknowledgements}

The authors would like to thank the members of the Chamberlain, Lalande, Graveley, and Levine labs for helpful discussions. This work was funded by $\mathrm{NIH/NICHD} \mathrm{grant} \mathrm{5R01HD068730-02} \mathrm{(S.J.C),} \mathrm{the} \mathrm{Raymond} \mathrm{and} \mathrm{Beverly} \mathrm{Sackler}$ Foundation (S.J.C), the Prutting fund (M.L.), and Autism Speaks (E.S.L.).

\section{Author details \\ ${ }^{1}$ Department of Genetics and Developmental Biology, University of Connecticut Health Center, 400 Farmington Avenue, Farmington, CT 06032, USA. ${ }^{2}$ Chromosome Core, Department of Molecular and Cell Biology and Department of Allied Health Sciences, University of Connecticut, 354 Mansfield Road, Storrs, CT 06269, USA. ${ }^{3}$ Department of Neuroscience, University of Connecticut Health Center, 263 Farmington Avenue, Farmington, CT 06030, USA. ${ }^{4}$ Department of Neurology, University of Tennessee Health Science Center, 855 Monroe Avenue, Suite 415, Memphis, TN 38163, USA. ${ }^{5}$ University of Connecticut Institute for Systems Genomics, Farmington, CT 06030, USA.}

Received: 24 March 2014 Accepted: 1 August 2014 Published: 20 August 2014

\section{References}

1. Marshall CR, Noor A, Vincent JB, Lionel AC, Feuk L, Skaug J, Shago M, Moessner R, Pinto D, Ren Y, Thiruvahindrapduram B, Fiebig A, Schreiber S,
Friedman J, Ketelaars CE, Vos YJ, Ficicioglu C, Kirkpatrick S, Nicolson R, Sloman L, Summers A, Gibbons CA, Teebi A, Chitayat D, Weksberg R, Thompson A, Vardy C, Crosbie V, Luscombe S, Baatjes R, et al: Structural variation of chromosomes in autism spectrum disorder. Am J Hum Genet 2008, 82:477-488.

2. Schroer RJ, Phelan MC, Michaelis RC, Crawford EC, Skinner SA, Cuccaro M, Simensen RJ, Bishop J, Skinner C, Fender D, Stevenson RE: Autism and maternally derived aberrations of chromosome 15q. Am J Med Genet 1998, 76:327-336.

3. Nishimura Y, Martin CL, Vazquez-Lopez A, Spence SJ, Alvarez-Retuerto Al, Sigman M, Steindler C, Pellegrini S, Schanen NC, Warren ST, Geschwind DH: Genome-wide expression profiling of lymphoblastoid cell lines distinguishes different forms of autism and reveals shared pathways. Hum Mol Genet 2007, 16:1682-1698.

4. Cook EH Jr, Lindgren V, Leventhal BL, Courchesne R, Lincoln A, Shulman C, Lord C, Courchesne E: Autism or atypical autism in maternally but not paternally derived proximal 15q duplication. Am J Hum Genet 1997, 60:928-934

5. Koochek M, Harvard C, Hildebrand MJ, Van Allen M, Wingert H, Mickelson E, Holden JJ, Rajcan-Separovic E, Lewis ME: 15q duplication associated with autism in a multiplex family with a familial cryptic translocation $\mathrm{t}$ $(14 ; 15)(q 11.2 ;$ 13.3) detected using array-CGH. Clin Genet 2006, 69:124-134.

6. Szatmari P, Paterson AD, Zwaigenbaum L, Roberts W, Brian J, Liu XQ Vincent JB, Skaug JL, Thompson AP, Senman L, Feuk L, Qian C, Bryson SE, Jones MB, Marshall CR, Scherer SW, Vieland VJ, Bartlett C, Mangin LV, Goedken R, Segre A, Pericak-Vance MA, Cuccaro ML, Gilbert JR, Wright HH, Abramson RK, Betancur C, Bourgeron T, Gillberg C, Leboyer M, et al: Mapping autism risk loci using genetic linkage and chromosomal rearrangements. Nat Genet 2007, 39:319-328.

7. Vorstman JA, Staal WG, Van Daalen E, Van Engeland H, Hochstenbach PF, Franke L: Identification of novel autism candidate regions through analysis of reported cytogenetic abnormalities associated with autism. Mol Psychiatry 2006, 11:18-28.

8. Urraca N, Cleary J, Brewer V, Pivnick EK, McVicar K, Thibert RL, Schanen NC, Esmer C, Lamport D, Reiter LT: The interstitial duplication 15q11.2-q13 syndrome includes autism, mild facial anomalies and a characteristic EEG signature. Autism Res 2013, 6:268-279.

9. Hogart A, Wu D, LaSalle JM, Schanen NC: The comorbidity of autism with the genomic disorders of chromosome 15q11.2-q13. Neurobiol Dis 2010, 38:181-191.

10. Trillingsgaard A, JR OS: Autism in Angelman syndrome: an exploration of comorbidity. Autism 2004, 8:163-174.

11. Rougeulle $\mathrm{C}$, Glatt $\mathrm{H}$, Lalande M: The Angelman syndrome candidate gene, UBE3A/E6-AP, is imprinted in brain. Nat Genet 1997, 17:14-15.

12. Kishino T, Lalande M, Wagstaff J: UBE3A/E6-AP mutations cause Angelman syndrome. Nat Genet 1997, 15:70-73.

13. Matsuura T, Sutcliffe JS, Fang P, Galjaard RJ, Jiang YH, Benton CS, Rommens JM, Beaudet AL: De novo truncating mutations in E6-AP ubiquitin-protein ligase gene (UBE3A) in Angelman syndrome. Nat Genet 1997, 15:74-77.

14. Smith SE, Zhou YD, Zhang G, Jin Z, Stoppel DC, Anderson MP: Increased gene dosage of Ube3a results in autism traits and decreased glutamate synaptic transmission in mice. Sci Trans/ Med 2011, 3:103ra197.

15. Schenck A, Bardoni B, Moro A, Bagni C, Mandel JL: A highly conserved protein family interacting with the fragile $X$ mental retardation protein (FMRP) and displaying selective interactions with FMRP-related proteins FXR1P and FXR2P. Proc Natl Acad Sci U S A 2001, 98:8844-8849.

16. Goytain A, Hines RM, El-Husseini A, Quamme GA: NIPA1(SPG6), the basis for autosomal dominant form of hereditary spastic paraplegia, encodes a functional Mg2+ transporter. J Biol Chem 2007, 282:8060-8068.

17. Svenstrup K, Moller RS, Christensen J, Budtz-Jorgensen E, Gilling M, Nielsen JE: NIPA1 mutation in complex hereditary spastic paraplegia with epilepsy. Eur J Neurol 2011, 18:1197-1199.

18. Chamberlain SJ, Chen PF, Ng KY, Bourgois-Rocha F, Lemtiri-Chlieh F, Levine ES, Lalande M: Induced pluripotent stem cell models of the genomic imprinting disorders Angelman and Prader-Willi syndromes. Proc Natl Acad Sci U S A 2010, 107:17668-17673.

19. Pankratz MT, Li XJ, Lavaute TM, Lyons EA, Chen X, Zhang SC: Directed neural differentiation of human embryonic stem cells via an obligated primitive anterior stage. Stem Cells 2007, 25:1511-1520. 
20. Germain ND, Banda EC, Becker S, Naegele JR, Grabel LB: Derivation and isolation of NKX2.1-positive basal forebrain progenitors from human embryonic stem cells. Stem Cells Dev 2013, 22:1477-1489.

21. Martins-Taylor K, Hsiao JS, Chen PF, Glatt-Deeley H, De Smith AJ, Blakemore Al, Lalande M, Chamberlain SJ: Imprinted expression of UBE3A in non-neuronal cells from a Prader-Willi syndrome patient with an atypical deletion. Hum Mol Genet 2013, 23:2364-2373.

22. Lieberman R, Levine ES, Kranzler HR, Abreu C, Covault J: Pilot study of iPS-derived neural cells to examine biologic effects of alcohol on human neurons in vitro. Alcohol Clin Exp Res 2012, 36:1678-1687.

23. Smibert P, Miura P, Westholm JO, Shenker S, May G, Duff MO, Zhang D, Eads BD, Carlson J, Brown JB, Eisman RC, Andrews J, Kaufman T, Cherbas P, Celniker SE, Graveley BR, Lai EC: Global patterns of tissue-specific alternative polyadenylation in Drosophila. Cell Rep 2012, 1:277-289.

24. Langmead B, Trapnell C, Pop M, Salzberg SL: Ultrafast and memoryefficient alignment of short DNA sequences to the human genome. Genome Biol 2009, 10:R25.

25. Trapnell C, Roberts A, Goff L, Pertea G, Kim D, Kelley DR, Pimentel H, Salzberg SL, Rinn JL, Pachter L: Differential gene and transcript expression analysis of RNA-seq experiments with TopHat and Cufflinks. Nat Protoc 2012, 7:562-578.

26. Takahashi K, Tanabe K, Ohnuki M, Narita M, Ichisaka T, Tomoda K, Yamanaka S: Induction of pluripotent stem cells from adult human fibroblasts by defined factors. Cell 2007, 131:861-872.

27. Sommer CA, Stadtfeld M, Murphy GJ, Hochedlinger K, Kotton DN, Mostoslavsky G: Induced pluripotent stem cell generation using a single lentiviral stem cell cassette. Stem Cells 2009, 27:543-549.

28. Jima DD, Zhang J, Jacobs C, Richards KL, Dunphy CH, Choi WW, Au WY, Srivastava G, Czader MB, Rizzieri DA, Lagoo AS, Lugar PL, Mann KP, Flowers CR, Bernal-Mizrachi L, Naresh KN, Evens AM, Gordon LI, Luftig M, Friedman DR, Weinberg JB, Thompson MA, Gill Jl, Liu Q, How T, Grubor V, Gao Y, Patel $\mathrm{A}, \mathrm{Wu} \mathrm{H}$, Zhu J, et al: Deep sequencing of the small RNA transcriptome of normal and malignant human $B$ cells identifies hundreds of novel microRNAs. Blood 2010, 116:e118-e127.

29. Herman JG, Graff JR, Myohanen S, Nelkin BD, Baylin SB: Methylationspecific PCR: a novel PCR assay for methylation status of $\mathrm{CpG}$ islands. Proc Natl Acad Sci U S A 1996, 93:9821-9826.

30. Kosaki K, McGinniss MJ, Veraksa AN, McGinnis WJ, Jones KL: Prader-Willi and Angelman syndromes: diagnosis with a bisulfite-treated methylation-specific PCR method. Am J Med Genet 1997, 73:308-313.

31. Johnson MA, Weick JP, Pearce RA, Zhang SC: Functional neural development from human embryonic stem cells: accelerated synaptic activity via astrocyte coculture. J Neurosci 2007, 27:3069-3077.

32. Chamberlain SJ, Brannan Cl: The Prader-Willi syndrome imprinting center activates the paternally expressed murine Ube3a antisense transcript but represses paternal Ube3a. Genomics 2001, 73:316-322.

33. Hogart A, Patzel KA, LaSalle JM: Gender influences monoallelic expression of ATP10A in human brain. Hum Genet 2008, 124:235-242.

34. Scoles HA, Urraca N, Chadwick SW, Reiter LT, Lasalle JM: Increased copy number for methylated maternal $15 q$ duplications leads to changes in gene and protein expression in human cortical samples. Mol Autism 2011, 2:19.

35. Baron CA, Tepper CG, Liu SY, Davis RR, Wang NJ, Schanen NC, Gregg JP. Genomic and functional profiling of duplicated chromosome 15 cell lines reveal regulatory alterations in UBE3A-associated ubiquitinproteasome pathway processes. Hum Mol Genet 2006, 15:853-869

36. Miller DM, Polansky DA, Thomas SD, Ray R, Campbell WW, Sanchez J, Koller CA: Mithramycin selectively inhibits transcription of G-C containing DNA. Am J Med Sci 1987, 294:388-394.

37. Blume SW, Snyder RC, Ray R, Thomas S, Koller CA, Miller DM: Mithramycin inhibits SP1 binding and selectively inhibits transcriptional activity of the dihydrofolate reductase gene in vitro and in vivo. J Clin Invest 1991, 88:1613-1621.

38. Kent WJ, Sugnet CW, Furey TS, Roskin KM, Pringle TH, Zahler AM, Haussler D: The human genome browser at UCSC. Genome Res 2002, 12:996-1006.

39. Sleiman SF, Langley BC, Basso M, Berlin J, Xia L, Payappilly JB, Kharel MK, Guo H, Marsh JL, Thompson LM, Mahishi L, Ahuja P, MacLellan WR, Geschwind DH, Coppola G, Rohr J, Ratan RR: Mithramycin is a geneselective Sp1 inhibitor that identifies a biological intersection between cancer and neurodegeneration. J Neurosci 2011, 31:6858-6870.
40. He Y, Casaccia-Bonnefil P: The Yin and Yang of $Y Y 1$ in the nervous system. J Neurochem 2008, 106:1493-1502.

41. Thomas MJ, Seto E: Unlocking the mechanisms of transcription factor YY1: are chromatin modifying enzymes the key? Gene 1999, 236:197-208.

42. Meguro-Horike M, Yasui DH, Powell W, Schroeder DI, Oshimura M, Lasalle JM, Horike S: Neuron-specific impairment of inter-chromosomal pairing and transcription in a novel model of human 15q-duplication syndrome. Hum Mol Genet 2011, 20:3798-3810.

43. Herzing LB, Cook EH Jr, Ledbetter DH: Allele-specific expression analysis by RNA-FISH demonstrates preferential maternal expression of UBE3A and imprint maintenance within 15q11-q13 duplications. Hum Mol Genet 2002, 11:1707-1718.

44. Kayashima T, Ohta T, Niikawa N, Kishino T: On the conflicting reports of imprinting status of mouse ATP10a in the adult brain: strainbackground-dependent imprinting? J Hum Genet 2003, 48:492-493. author reply 494

45. Kuhnle S, Kogel U, Glockzin S, Marquardt A, Ciechanover A, Matentzoglu K, Scheffner M: Physical and functional interaction of the HECT ubiquitinprotein ligases E6AP and HERC2. J Biol Chem 2011, 286:19410-19416.

46. Kleinjan DA, Van Heyningen V: Long-range control of gene expression: emerging mechanisms and disruption in disease. Am J Hum Genet 2005, 76:8-32.

47. Phillips-Cremins JE, Sauria ME, Sanyal A, Gerasimova TI, Lajoie BR, Bell JS, Ong CT, Hookway TA, Guo C, Sun Y, Bland MJ, Wagstaff W, Dalton S, McDevitt TC, Sen R, Dekker J, Taylor J, Corces VG: Architectural protein subclasses shape 3D organization of genomes during lineage commitment. Cell 2013, 153:1281-1295.

48. Voineagu I, Wang $X$, Johnston $P$, Lowe JK, Tian $Y$, Horvath S, Mill J, Cantor RM, Blencowe BJ, Geschwind DH: Transcriptomic analysis of autistic brain reveals convergent molecular pathology. Nature 2011, 474:380-384.

49. Andrusiak MG, Vandenbosch R, Park DS, Slack RS: The retinoblastoma protein is essential for survival of postmitotic neurons. J Neurosci 2012, 32:14809-14814.

50. Herrup K, Yang Y: Cell cycle regulation in the postmitotic neuron: oxymoron or new biology? Nat Rev Neurosci 2007, 8:368-378.

51. Lee SY, Ramirez J, Franco M, Lectez B, Gonzalez M, Barrio R, Mayor U: Ube3a, the E3 ubiquitin ligase causing Angelman syndrome and linked to autism, regulates protein homeostasis through the proteasomal shuttle Rpn10. Cell Mol Life Sci 2013, 71:2747-2758.

52. Brown JH, Kennedy BJ: Mithramycin in the Treatment of Disseminated Testicular Neoplasms. N Engl J Med 1965, 272:111-118.

53. Kennedy BJ, Brown JH, Yarbro JW: Mithramycin (NSC-24559) therapy for primary glioblastomas. Cancer Chemother Rep 1965, 48:59-63.

54. Singer FR, Neer RM, Murray TM, Keutmann HT, Deftos LJ, Potts JT Jr: Mithramycin treatment of intractable hypercalcemia due to parathyroid carcinoma. N Engl J Med 1970, 283:634-636.

doi:10.1186/2040-2392-5-44

Cite this article as: Germain et al:: Gene expression analysis of human induced pluripotent stem cell-derived neurons carrying copy number variants of chromosome 15q11-q13.1. Molecular Autism 2014 5:44.

\section{Submit your next manuscript to BioMed Central and take full advantage of:}

- Convenient online submission

- Thorough peer review

- No space constraints or color figure charges

- Immediate publication on acceptance

- Inclusion in PubMed, CAS, Scopus and Google Scholar

- Research which is freely available for redistribution 